\title{
FEASIBILITY OF USING PLANTS TO ASSIST IN THE REMEDIATION OF HEAVY METAL CONTAMINATION AT J-FIELD, ABERDEEN PROVING GROUND, MARYLAND
}

\author{
FINAL REPORT \\ 3 November 1995 \\ J.D. Jastrow \\ Environmental Research Division \\ Argonne National Laboratory
}

AT RTIVED

MAR 271996

OSTI

\section{Introduction}

Most remedial technologies currently being used at hazardous waste sites (e.g., containment, excavation, soil washing, or incineration) are expensive. Further, in some locations technologies involving excavation could increase off-site releases of hazardous materials by destabilizing the site. Thus, interest in the development of in situ bioremediation technologies has grown substantially over the last decade. The idea of phytoremediation (i.e., using plants to clean up toxic wastes) is generating increasing attention from scientists, industry, and government agencies. The attractiveness of phytoremediation stems from its potential (1) to be less expensive than technologies involving the human engineering costs of soil manipulation, and (2) to initiate simultaneously both the clean up of hazardous materials and site restoration (Stomp et al., 1993).

The ability of plants to colonize and tolerate metalliferous soils has been known for some time (Antonovics et al., 1971). Metal tolerant plants have evolved physiological mechanisms that generally do not suppress metal uptake but rather result in internal detoxification via two basic strategies (Baker, 1981). In accumulators, metals are concentrated in aboveground parts regardless of soil concentrations. In contrast, excluders maintain aboveground concentrations that are constant and low over a wide range of soil concentrations by restricting transport from roots to shoots. Thus, the two strategies differ in their sites of detoxification (i.e., roots in excluders and shoots in accumulators) (Baker, 1981). However, heavy metal availability, uptake, and accumulation at a given site may be largely influenced by a variety of factors ranging from

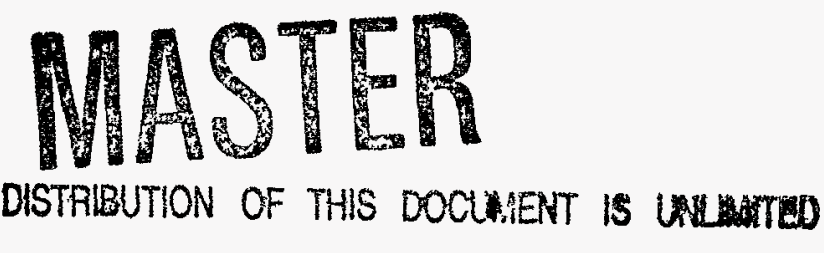




\section{DISCLAMMER}

Portions of this document may be illegible in electronic image products. Images are produced from the best available original document. 
the form of metal contamination to edaphic factors affecting soil sorption capacities (Miller et al, 1975; Barry and Clark, 1978).

Most phytoremediation strategies will likely require metal tolerant plants of the accumulator type. The accumulation of metal contaminants in aboveground tissue facilitates plant harvest and, thus, cost-effective removal and disposal of the accumulated contaminants. Further, the identification or selection of "hyperaccumulator" plants (Baker et al., 1988) and the potential for similar genetically engineered capabilities (Stomp et al., 1993) offer some promise for the feasibility of such phytoremediation technologies. However, successful phytoremediation will also require species that (1) produce significant amounts of aboveground biomass, and (2) possess root systems capable of proliferating throughout and, thereby, extracting contaminants from the entire volumetric extent of the contaminated area.

The purpose of this project was to investigate the potential for using plants to remediate J-Field soils contaminated with heavy metals. Phragmites australis, one of the dominant species in the Toxic Burning Pits (TBP) area and other contaminated sites within J-Field, appears to be both tolerant of heavy metal contaminated soil conditions and capable of producing large amounts of biomass. Consequently, this project has concentrated on characterizing heavy metal accumulation by Phragmites australis growing in the TBP area relative to soil concentrations and availabilities. This type of information is necessary to determine the feasibility of using this species to assist in the remediation of metal contaminated soils at J-Field.

\section{Materials and Methods}

Thirty sampling locations ( 20 from upland areas and 10 from the marsh) were marked for sample collection (Fig. 1). The upland sites were selected on the basis of previous soil characterizations to provide a range of heavy metal concentrations (from high levels in the pushout area to relatively low levels in areas less directly affected by TBP activities, e.g., sites 17-20). The marsh areas were selected along a transect accessible by the boardwalks and roughly parallel to the marsh edge. In July 1994, aboveground parts of Phragmites australis were clipped at the soil surface and removed from each of these locations. Belowground parts were collected by digging beneath the clipped stems with an 18-cm wide spade to remove a block of soil to a depth 


\section{3}

of $15 \mathrm{~cm}$. Soil from in and around the belowground plant samples was also collected to a depth of $15 \mathrm{~cm}$ at each location. In December 1994, stems of Phragmites australis were again clipped and removed from the same 30 locations. No soil samples were taken at that time. The purpose of the second sampling was to determine whether plant uptake of heavy metals remains relatively constant throughout the growing season or lags behind the rate of plant biomass accumulation over the course of the growing season resulting in lower heavy metal concentrations in the plant tissue.

After samples were shipped to Argonne, aboveground plant tissue was washed with deionized water and separated into stems, leaves, and flowers/seeds (when present).

Belowground plant tissue was thoroughly washed to remove the surrounding soil and separated into roots and rhizomes. Roots that were not attached to the Phragmites rhizomes were discarded. All plant tissue samples were dried at $65^{\circ} \mathrm{C}$, weighed, and ground with a stainless steel Wiley mill. Soil samples were air dried and, after removing roots and other large pieces of organic debris, gently crushed to pass through a $2-\mathrm{mm}$ sieve.

Plant tissue and soil samples (including duplicate samples created for QA/QC purposes by subsampling the original samples) were sent to the Research Extension Analytical Laboratory (REAL) at Ohio State University for nutrient and heavy metal (total concentration) analysis. Soil samples were also sent to the University of Wisconsin-Extension Soil and Plant Analysis Laboratory for determination of available (DTPA-extractable) heavy metals. Selected soil and plant samples were also analyzed at Wisconsin for nutrients and total heavy metals to ensure that results from the two labs were comparable. Both labs follow standard QA/QC procedures, use NIST traceable standards and other internal reference standards, and participate in external sample exchange and analysis programs with other laboratories.

Standard methods for plant and soil analysis (Page et al., 1982) or adaptations of these methods were used by the two labs. At the REAL lab, plant tissue and soil samples were digested in perchloric acid and analyzed for "total" heavy metals by inductively coupled plasma (ICP) optical emission spectroscopy. At the Wisconsin lab, soil samples were digested in 6:1 nitric/perchloric acid for "total" heavy metals and extracted by the diethylene triamine pentaacetic acid (DTPA) method for "available" heavy metals. Digests and extracts were analyzed by ICP optical emission spectroscopy. Plant tissue samples were digested with 
5:3 nitric acid/hydrogen peroxide and analyzed for mineral nutrients by ICP optical emission spectroscopy and for heavy metals by ICP mass spectroscopy. At both labs, available P was determined by the Bray $\mathrm{P}_{1}$ method. Available $\mathrm{K}$ was determined on the Bray $\mathrm{P}_{1}$ extracts at Wisconsin and on ammonium acetate extracts at the REAL lab. Exchangeable $\mathrm{Ca}$ and $\mathrm{Mg}$ were determined on ammonium acetate extracts at both labs. Cation exchange capacity was determined at the REAL lab by the ammonium acetate method. Organic matter was determined by the combustion method at both labs. Total $\mathrm{N}$ was determined at Wisconsin by the semi-micro Kjeldahl procedure. Electrical conductivity and $\mathrm{pH}$ were determined by standard methods on water extracts.

\section{Results and Discussion}

All analytical results from both labs are presented in the Appendix. Visual analysis of the data indicated that the variability between duplicate samples analyzed at both labs was similar to the variability between duplicates analyzed within the same laboratory. Most duplicates were reasonably comparable. Data variability was probably due more to the inherent heterogeneity of the samples or to the variable forms of contaminants within the samples than to analytical errors within or between laboratories. Thus, valid comparisons between DTPA-extractable metals determined at Wisconsin can be made to total metals determined mostly at the REAL lab. Also, all replicate analyses were averaged to give a single value for each sampling site prior to statistical analysis of the data.

Although this report will focus on $\mathrm{Pb}$ because of the emphasis on it in remediation planning for the TBP site, examination of the data revealed that $\mathrm{Cu}$ and $\mathrm{Zn}$ (and possibly $\mathrm{Cr}$ depending on the valence state) are present in the soil at concentrations that can be toxic to many plant species (Chapman, 1966). Among the other edaphic factors that may affect plant growth at the site are $\mathrm{P}$ concentrations that range from very low (deficient) to extremely high (potentially toxic) and relatively low $\mathrm{N}$ concentrations.

Total $\mathrm{Pb}$ in the soil ranged from a low of $82 \mu \mathrm{g} / \mathrm{g}$ at site 19 located in the relatively uncontaminated area on the edge of the forest uphill from the pits to a high of $>41,000 \mu \mathrm{g} / \mathrm{g}$ at site 2 in the push-out area (Table 1). Samples from the marsh were relatively constant at about 


\section{5}

$750 \mu \mathrm{g} / \mathrm{g}$. Available (DTPA-extractable) $\mathrm{Pb}$ in the soil ranged from $20 \mu \mathrm{g} / \mathrm{g}$ at site 15 to about $1400 \mu \mathrm{g} / \mathrm{g}$ at site 4 . Available $\mathrm{Pb}$ in the marsh samples were again relatively constant with a mean of $233 \mu \mathrm{g} / \mathrm{g}$.

The sites with the lowest and highest values for available $\mathrm{Pb}$ did not correspond to the sites with the lowest and highest total $\mathrm{Pb}$ concentrations, however available and total $\mathrm{Pb}$ were highly correlated $(\mathrm{r}=0.75 ; \mathrm{P}<0.001)$ in the upland area because of its wide range in soil $\mathrm{Pb}$ contents. In the marsh, available and total $\mathrm{Pb}$ were not significantly correlated. The proportion of total $\mathrm{Pb}$ extracted by the DTPA procedure ranged from 0.01 at site 2 to 0.45 at site 26 and was generally higher in the marsh than in the upland area (Table 1). The proportion of available $\mathrm{Pb}$ was negatively associated with total $\mathrm{Pb}$ as well as available $\mathrm{Pb}(\mathrm{r}=-0.64$ and -0.58 , respectively; $\mathrm{P}<0.01)$ in the upland area, but it was positively correlated with available $\mathrm{Pb}(\mathrm{r}=0.66 ; \mathrm{P}<0.05)$ in the marsh.

The highest concentration of $\mathrm{Pb}$ found in aboveground plant tissue during July was $23 \mu \mathrm{g} / \mathrm{g}$ in the leaves of the plant located at site 3 , which had the second highest concentration of available soil $\mathrm{Pb}$. In December, leaves from plants at this location contained $110 \mu \mathrm{g} / \mathrm{g} \mathrm{Pb}$. However, most plants from both sampling times had considerably lower concentrations of $\mathrm{Pb}$ in their aboveground tissue (Tables 2 and 3). Mean $\mathrm{Pb}$ concentrations in the entire shoot were greater at the end of the growing season than in July. Shoot $\mathrm{Pb}$ concentrations were also higher in the upland area than in the marsh, partly because mean concentrations of $\mathrm{Pb}$ in the soil were greater in the upland area and partly because of the diluting effects of greater phytomass production in the marsh.

The concentration of $\mathrm{Pb}$ in belowground tissue was considerably greater than in aboveground tissue (Tables 2 and 3), suggesting that Phragmites tolerates high soil $\mathrm{Pb}$ concentrations by using an excluder (Baker, 1981) or an avoidance (Shann, 1995) strategy. Most $\mathrm{Pb}$ taken up by the plant is apparently sequestered in belowground tissue, possibly in the cell walls of conductive tissue and in root intercellular spaces. Gries and Garbe (1989) suggested that $\mathrm{Pb}$ and other heavy metals may be bound as co-precipitates in iron hydroxide plaques on root surfaces, pointing out that the binding of heavy metals as oxides can be an uptake avoidance mechanism in flooded (reduced) root environments. Concentrations were highest in the rhizomes from site $4(801 \mu \mathrm{g} / \mathrm{g})$, which also had the highest available soil $\mathrm{Pb}$ and in the roots 
from site $2(3400 \mu \mathrm{g} / \mathrm{g})$, which had the highest total soil $\mathrm{Pb}$. Again, most plants had considerably lower $\mathrm{Pb}$ concentrations in their belowground tissue (Tables 2 and 3 ).

Plant:soil concentration ratios were calculated for the marsh and upland areas (Table 4). Lead concentration ratios for shoots were very small and were at least an order of magnitude lower than for roots. Rhizome concentration ratios were intermediate. These ratios, to a certain extent, assist in comparisons of relative uptake by plants growing on soils with different $\mathrm{Pb}$ contents and also emphasize how little $\mathrm{Pb}$ is translocated to shoot tissue.

Shoot and belowground tissue concentrations of $\mathrm{Pb}$ were highly correlated with both total and available soil $\mathrm{Pb}$ concentrations in the upland area (correlation coefficients were generally greater than $0.80 ; \mathrm{P}<0.001$ ). In the marsh, however, there were no significant relationships between soil $\mathrm{Pb}$ concentrations and plant tissue concentrations above or below ground in July. In December, shoot concentrations were negatively correlated with total soil $\mathrm{Pb}(\mathrm{r}=-0.68 ; \mathrm{P}<0.05)$ and available $\mathrm{Pb}(\mathrm{r}=-0.56 ; \mathrm{P}<0.10)$. This finding suggests that other edaphic factors (Table 5) in the marsh (e.g., the higher organic matter content and/or cation exchange capacity) are influencing $\mathrm{Pb}$ availability and that this effect is not being measured under these conditions by the DTPA extraction method for estimating $\mathrm{Pb}$ availability. In fact, available (DTPA extractable) $\mathrm{Pb}$ in the marsh was negatively correlated with organic matter $(\mathrm{r}=-0.81 ; \mathrm{P}<0.01)$, available $\mathrm{P}$ $(r=-0.68 ; P<0.10)$, cation exchange capacity $(r=-0.57 ; P<0.10)$, and electrical conductivity $(\mathrm{r}=-0.81 ; \mathrm{P}<0.01)$.

Closer examination of the relationships between plant tissue $\mathrm{Pb}$ and soil $\mathrm{Pb}$ in the upland areas, however, revealed that the strong correlations were due primarily to the influence of sites with very high soil $\mathrm{Pb}$ concentrations as illustrated, for example, in Fig. 2. Yet, phytoremediation would not likely be used as a remediation strategy at the J-Field Site until total $\mathrm{Pb}$ concentrations in the soil were reduced by other technologies to $500 \mu \mathrm{g} / \mathrm{g}$ or less. Because only six of the sampled sites had total soil $\mathrm{Pb}$ concentrations that were $<500 \mu \mathrm{g} / \mathrm{g}$, all further evaluations of the feasibility of using Phragmites for phytoremediation at the site were based on the 17 sites with total soil $\mathrm{Pb}$ concentrations $<1000 \mu \mathrm{g} / \mathrm{g}$, which gives more realistic assessments than can be made by using all 30 sites. This subset of sites provides a mean total soil $\mathrm{Pb}$ concentration of $534 \mu \mathrm{g} / \mathrm{g}$ (Table 6) and includes nine sites from the marsh and eight from the upland areas. Plant tissue concentrations and plant:soil concentration ratios for this subset of 


\section{7}

sites are characterized in Table 7 and 8 . After deleting those sites with higher soil $\mathrm{Pb}$ from the dataset, plant tissue concentrations were, indeed, no longer significantly correlated with total or available soil $\mathrm{Pb}$ concentrations (e.g., Fig. 3).

Total aboveground uptake of $\mathrm{Pb}$ by the sampled plants was calculated by multiplying shoot $\mathrm{Pb}$ concentrations by sample dry weights (Table 9). Lead uptake was about 3 to 4 times greater for marsh plants than for plants growing in upland areas, in part because $\mathrm{Pb}$ concentrations were slightly higher in marsh plants but mostly because plants were larger in the marsh. Uptake was not significantly correlated with total soil $\mathrm{Pb}$ but was positively correlated with available $\mathrm{Pb}$ in both July and December (Table 10). Aboveground $\mathrm{Pb}$ uptake was also negatively correlated with soil $\mathrm{pH}$ but was positively correlated with soil organic matter, cation exchange capacity, and electrical conductivity. However, it is unclear whether these relationships are truly indicative of the effects of the edaphic factors on $\mathrm{Pb}$ availability because the relationships are being driven, to a large extent, by the fact that $\mathrm{Pb}$ uptake is highest in the marsh, which also has higher organic matter, cation exchange capacity, and electrical conductivity as well as lower $\mathrm{pH}$ than the upland areas.

Although $\mathrm{Pb}$ uptake was much greater at the end of the growing season, the values presented in Table 9 for December are underestimates of potential $\mathrm{Pb}$ uptake because most samples had few if any leaves remaining on the stems at the time of sampling. By making several assumptions, a better estimate of potential $\mathrm{Pb}$ uptake by Phragmites shoots was calculated (Table 11). Although the assumption that the biomass of the sampled area can be used to project average Phragmites production over a large area may result in an overestimate, the values presented in Table 11 represent a realistic estimate of the best potential aboveground $\mathrm{Pb}$ uptake that can be expected for Phragmites without any soil manipulations to enhance $\mathrm{Pb}$ availability.

\section{Conclusions}

The estimates of potential $\mathrm{Pb}$ uptake on an areal basis do not appear to be high enough to make harvesting of aboveground Phragmites tissue a viable phytoremediation strategy unless (1) uptake can be enhanced by soil amendments or (2) it is not necessary to remove all of the $\mathrm{Pb}$ 
found in the soil. In the latter case, more information is needed to assess (1) what proportion of the total soil $\mathrm{Pb}$ content is available for plant uptake at any given time, (2) what the supply rate to the available pool is, and (3) how this rate could be manipulated. In addition, if some method of harvesting belowground tissue was physically, economically, and biologically feasible, it might allow a phytoremediation scheme employing Phragmites to remove greater amounts of $\mathrm{Pb}$ at a faster rate. However, the amount of root and rhizome tissue per unit volume of soil is probably relatively small compared to aboveground tissue (particularly in areas where soil $\mathrm{Pb}$ concentrations are high), making the feasibility of this approach unlikely for upland areas. In a marsh situation (as proposed for creation in the TBP area after physical removal and disposal of soil contaminated to some set concentration), belowground biomass production should be greater and harvesting of belowground tissue might be easier.

To assist in the decision-making process, the following simple calculations may prove useful. Assuming that (1) soil $\mathrm{Pb}$ concentrations will not exceed $500 \mu \mathrm{g} / \mathrm{g}$ after other technologies have been employed, (2) phytoremediation needs to effectively reduce soil $\mathrm{Pb}$ concentrations to only $300 \mu \mathrm{g} / \mathrm{g}$ to be considered successful, (3) soil bulk density is $2 \mathrm{~g} / \mathrm{cm}^{3}$, and (4) the effective rooting depth for $\mathrm{Pb}$ removal by Phragmites is $30 \mathrm{~cm}$, the soil $\mathrm{Pb}$ load to be removed may be estimated as

$(200 \mu \mathrm{g} \mathrm{Pb} / \mathrm{g}$ soil $) \times\left(2 \mathrm{~g} \mathrm{soil} / \mathrm{cm}^{3}\right.$ soil $) \times\left(3 \times 10^{5} \mathrm{~cm}^{3} / \mathrm{m}^{2}\right.$ to $\left.30 \mathrm{~cm}\right)=120 \mathrm{~g} \mathrm{~Pb} / \mathrm{m}^{2}$ to $30 \mathrm{~cm}$. to be removed to be removed

At a potential $\mathrm{Pb}$ uptake rate for Phragmites of about $0.05 \mathrm{~g} / \mathrm{m}^{2} / \mathrm{yr}$ (based on values in Table 11 for the marsh sites), it would take $120 / 0.05$ or 2400 years to reduce total soil $\mathrm{Pb}$ concentrations in the surface $30 \mathrm{~cm}$ to $300 \mu \mathrm{g} / \mathrm{g}$. Conversely, it would require annual $\mathrm{Pb}$ removal rates of $6 \mathrm{~g} / \mathrm{m}^{2} / \mathrm{yr}$ to reach this soil concentration in 20 years or $2.4 \mathrm{~g} / \mathrm{m}^{2} / \mathrm{yr}$ to reach it in 50 years. Assuming that annual aboveground Phragmites production could possibly reach $5 \mathrm{~kg} / \mathrm{m}^{2}$ (calculated from data in Table 11), these removal rates would require average $\mathrm{Pb}$ tissue concentrations of 1200 and $480 \mu \mathrm{g} / \mathrm{g}$ in aboveground tissue for 20 and 50 year projects, respectively. As noted in Table 2 , the highest shoot tissue concentration measured in this study was $48.4 \mu \mathrm{g} / \mathrm{g}$.

Thus, it appears that tissue concentrations must be increased by at least an order of magnitude while at the same time maintaining the same high level of biomass production in 
order to effectively phytoremediate the site within a reasonable time frame, unless removal of even less than $200 \mu \mathrm{g} / \mathrm{g}$ of soil $\mathrm{Pb}$ is acceptable. It is impossible to say, based on this study, whether other measures such as belowground phytomass harvesting, soil manipulations, or the use of another plant species would help to achieve such a goal.

\section{Literature Cited}

Antonovics, J., A.D. Bradshaw, and R.G. Turner. 1971. Heavy metal tolerance in plants. Adv. Ecol. Res. 7:1-85.

Baker, A.J.M. 1981. Accumulators and excluders -- Strategies in the response of plants to heavy metals. J. Plant Nutr. 3:643-654.

Baker, A., R. Brooks, and R. Reeves. 1988. Growing for gold...and copper...and zinc. New Sci. March 10(p. 44-48).

Barry, S.A.S., and S.C. Clark. 1978. Problems of interpreting the relationship between the amounts of lead and zinc in plants and soil on metalliferous wastes. New Phytol. 81:773783.

Chapman, H.D. (ed.). 1966. Diagnostic Criteria for Plants and Soils. University of California, Division of Agricultural Science, Berkeley. 793 pp.

Gries, C., and D. Garbe. 1989. Biomass, and nitrogen, phosphorus and heavy metal content of Phragmites australis during the third growing season in a root zone waste water treatment. Arch. Hydrobiol. 117:97-105.

Miller, J.E., J.J. Hassett, and D.E. Koeppe. 1975. The effect of soil properties and extractable lead levels on lead uptake by soybeans. Comm. Soil Sci. Plant Anal. 6:339-347.

Page, A.L., R.H. Miller, and D.R. Keeney (eds.) 1982. Methods of Soil Analysis. Part 2. 2nd ed. Agronomy No. 9, Am. Soc. Agron., Soil Sci. Soc. Am., Madison, WI.

Shann, J.R. 1995. The role of plants and plant/microbial systems in the reduction of exposure. Environ. Health Perspectives. 103 (Supplement 5):13-15.

Stomp, A.-M., K.-H. Han, S. Wilbert, and M.P. Gordon. 1993. Genetic improvement of tree species for remediation of hazardous wastes. In Vitro Cell. Dev. Biol. 29:227-232. 


\begin{tabular}{|c|c|c|c|c|c|}
\hline Sample location & Variable & $\begin{array}{l}\text { Mean } \\
(\mu \mathrm{g} / \mathrm{g})\end{array}$ & $\begin{array}{c}\text { SE } \\
(\mu \mathrm{g} / \mathrm{g})\end{array}$ & $\begin{array}{c}\text { Minimum } \\
(\mu \mathrm{g} / \mathrm{g})\end{array}$ & $\begin{array}{c}\text { Maximum } \\
(\mu \mathrm{g} / \mathrm{g})\end{array}$ \\
\hline \multirow{3}{*}{$\begin{array}{c}\text { All sites } \\
n=30\end{array}$} & Total $\mathrm{Pb}$ & 5751 & 1983 & 82 & 41414 \\
\hline & Available $\mathrm{Pb}$ & 343 & 69 & 20 & 1408 \\
\hline & Proportion avail. & 0.19 & 0.02 & 0.01 & 0.45 \\
\hline \multirow{3}{*}{$\begin{array}{l}\text { Upland sites } \\
n=20\end{array}$} & Total $\mathrm{Pb}$ & 8249 & 2831 & 82 & 41414 \\
\hline & Available $\mathrm{Pb}$ & 399 & 101 & 20 & 1408 \\
\hline & Proportion avail. & 0.13 & 0.02 & 0.01 & 0.37 \\
\hline \multirow{3}{*}{$\begin{array}{c}\text { Marsh sites } \\
n=10\end{array}$} & Total $\mathrm{Pb}$ & 755 & 65 & 445 & 1258 \\
\hline & Available $\mathrm{Pb}$ & 233 & 19 & 140 & 319 \\
\hline & Proportion avail. & 0.32 & 0.03 & 0.20 & 0.45 \\
\hline
\end{tabular}

\begin{tabular}{|c|c|c|c|c|c|}
\hline Sample date & Plant part & $\begin{array}{l}\text { Mean } \\
(\mu \mathrm{g} / \mathrm{g})\end{array}$ & $\begin{array}{c}\text { SE } \\
(\mu \mathrm{g} / \mathrm{g})\end{array}$ & $\begin{array}{c}\text { Minimum } \\
(\mu \mathrm{g} / \mathrm{g})\end{array}$ & $\begin{array}{c}\text { Maximum } \\
(\mu \mathrm{g} / \mathrm{g})\end{array}$ \\
\hline \multirow{5}{*}{ July } & Stems & 8.4 & 1.2 & 3.0 & 21.8 \\
\hline & Leaves & 8.4 & 1.3 & 1.0 & 23.4 \\
\hline & Shoots ${ }^{\mathrm{a}}$ & 8.5 & 1.1 & 3.5 & 22.5 \\
\hline & Rhizomes & 140.4 & 46.4 & 5.8 & 801.1 \\
\hline & Roots $^{b}$ & 571.7 & 284.4 & 41.4 & 3400.3 \\
\hline \multirow[t]{4}{*}{ December } & Stems & 11.1 & 2.9 & 1.0 & 51.6 \\
\hline & Leaves $^{\mathrm{c}}$ & 20.3 & 7.5 & 3.9 & 109.7 \\
\hline & Flowers/seeds $^{\mathrm{d}}$ & 7.9 & 1.5 & 4.7 & 20.2 \\
\hline & Shoots $^{\mathrm{a}}$ & 11.3 & 2.7 & 0.9 & 48.4 \\
\hline \multicolumn{6}{|c|}{$\begin{array}{l}\text { a Value for each sampling site was a weighted average (based on tissue mass) of concentration } \\
\text { data for stems and leaves (July) and stems, leaves, and seeds (December). } \\
b n=12 \\
{ }^{c} n=16 \\
{ }^{d} n=10\end{array}$} \\
\hline
\end{tabular}


Table 3. Pb concentrations in plant tissue sampled in July and December for marsh sites $(\mathrm{n}=10)$

\begin{tabular}{|l|l|c|c|c|c|}
\hline Sample date & \multicolumn{1}{|c|}{ Plant part } & $\begin{array}{c}\text { Mean } \\
(\mu \mathrm{g} / \mathrm{g})\end{array}$ & $\begin{array}{c}\text { SE } \\
(\mu \mathrm{g} / \mathrm{g})\end{array}$ & $\begin{array}{c}\text { Minimum } \\
(\mu \mathrm{g} / \mathrm{g})\end{array}$ & $\begin{array}{c}\text { Maximum } \\
(\mu \mathrm{g} / \mathrm{g})\end{array}$ \\
\hline \multirow{5}{*}{ July } & Stems & 4.7 & 0.5 & 2.8 & 7.8 \\
\cline { 2 - 6 } & Leaves & 4.9 & 0.7 & 2.8 & 8.4 \\
\cline { 2 - 6 } & Shoots & 4.7 & 0.4 & 2.8 & 6.4 \\
\cline { 2 - 6 } & Rhizomes & 45.1 & 21.5 & 5.4 & 232.2 \\
\cline { 2 - 6 } & Roots & 128.8 & 38.3 & 21.5 & 338.8 \\
\hline \multirow{5}{*}{ December } & & & & & 14.3 \\
\cline { 2 - 6 } & Stems & 8.3 & 1.0 & 3.2 & 9.1 \\
\cline { 2 - 6 } & Leaves $^{\mathrm{a}}$ & 6.7 & 2.4 & 4.3 & 3.4 \\
\cline { 2 - 6 } & Flowers/seeds $^{\mathrm{b}}$ & 3.4 & 0.0 & 3.4 & 13.3 \\
\cline { 2 - 6 } & Shoots $^{\mathrm{a}}$ & 7.7 & 0.9 & 3.0 & \\
\hline
\end{tabular}

${ }^{\mathrm{a}}$ Value for each sampling site was a weighted average (based on tissue mass) of concentration data for stems and leaves (July) and stems, leaves, and seeds (December).

${ }^{\mathrm{b}} \mathrm{n}=2$

Table 4. Plant:soil $\mathrm{Pb}$ concentration ratios for plants sampled in July and December on upland $(n=20)$ and marsh $(n=10)$ sites

\begin{tabular}{|c|c|c|c|c|c|}
\hline Sample date & Plant part & Mean & $\mathrm{SE}$ & Minimum & Maximum \\
\hline \multicolumn{6}{|l|}{ Upland sites } \\
\hline \multirow[t]{3}{*}{ July } & Shoots & 0.011 & 0.003 & 0.0003 & 0.056 \\
\hline & Rhizomes & 0.047 & 0.012 & 0.008 & 0.157 \\
\hline & Roots $^{\mathrm{a}}$ & 0.281 & 0.077 & 0.023 & 0.899 \\
\hline December & Shoots & 0.012 & 0.004 & 0.0003 & 0.065 \\
\hline \multicolumn{6}{|l|}{ Marsh sites } \\
\hline \multirow[t]{3}{*}{ July } & Shoots & 0.007 & 0.001 & 0.003 & 0.013 \\
\hline & Rhizomes & 0.062 & 0.030 & 0.007 & 0.330 \\
\hline & Roots & 0.183 & 0.065 & 0.029 & 0.710 \\
\hline December & Shoots & 0.011 & 0.002 & 0.002 & 0.024 \\
\hline
\end{tabular}




\begin{tabular}{|c|c|c|c|c|c|}
\hline Sample Location & Variable & Mean & $\mathrm{SE}$ & Minimum & Maximum \\
\hline \multirow{5}{*}{$\begin{array}{l}\text { Upland sites } \\
n=20\end{array}$} & $\mathrm{pH}$ & 7.26 & 0.20 & 5.95 & 8.65 \\
\hline & Organic matter (\%) & 2.43 & 0.24 & 0.75 & 4.90 \\
\hline & Available P $(\mu \mathrm{g} / \mathrm{g})$ & 101 & 44 & 2 & 884 \\
\hline & $\begin{array}{l}\text { Cation exchange } \\
\text { capacity (meq) }\end{array}$ & 6.5 & 0.7 & 2 & 13 \\
\hline & $\begin{array}{l}\text { Electrical } \\
\text { conductivity (dS/m) }\end{array}$ & 0.26 & 0.06 & 0.05 & 1.08 \\
\hline \multirow{5}{*}{$\begin{array}{l}\text { Marsh sites } \\
n=10\end{array}$} & $\mathrm{pH}$ & 5.73 & 0.14 & 5.30 & 6.80 \\
\hline & Organic matter $(\%)$ & 13.14 & 1.72 & 7.40 & 22.50 \\
\hline & Available P $(\mu \mathrm{g} / \mathrm{g})$ & 105 & 16 & 49 & 204 \\
\hline & $\begin{array}{l}\text { Cation exchange } \\
\text { capacity (meq) }\end{array}$ & 18.9 & 1.4 & 15 & 29 \\
\hline & $\begin{array}{l}\text { Electrical } \\
\text { conductivity }(\mathrm{dS} / \mathrm{m})\end{array}$ & 3.45 & 0.67 & 1.50 & 8.45 \\
\hline
\end{tabular}

Table 6. Total and available (DTPA-extractable) $\mathrm{Pb}$ concentrations in soil and the proportion of available $\mathrm{Pb}$ (available $\mathrm{Pb}$ divided by total $\mathrm{Pb}$ ) for sites where total $\mathrm{Pb}$ in soil is $<1000 \mu \mathrm{g} / \mathrm{g}$ $(n=17)$

\begin{tabular}{|l|c|c|c|c|}
\hline \multicolumn{1}{|c|}{ Variable } & $\begin{array}{c}\text { Mean } \\
(\mu \mathrm{g} / \mathrm{g})\end{array}$ & $\begin{array}{c}\text { SE } \\
(\mu \mathrm{g} / \mathrm{g})\end{array}$ & $\begin{array}{c}\text { Minimum } \\
(\mu \mathrm{g} / \mathrm{g})\end{array}$ & $\begin{array}{c}\text { Maximum } \\
(\mu \mathrm{g} / \mathrm{g})\end{array}$ \\
\hline Total $\mathrm{Pb}$ & 534 & 63 & 82 & 848 \\
\hline Available $\mathrm{Pb}$ & 147 & 25 & 20 & 319 \\
\hline Proportion avail. & 0.27 & 0.03 & 0.06 & 0.45 \\
\hline
\end{tabular}




\begin{tabular}{|c|c|c|c|c|c|}
\hline Sample date & Plant part & $\begin{array}{l}\text { Mean } \\
(\mu \mathrm{g} / \mathrm{g})\end{array}$ & $\begin{array}{c}\mathrm{SE} \\
(\mu \mathrm{g} / \mathrm{g})\end{array}$ & $\begin{array}{c}\text { Minimum } \\
(\mu \mathrm{g} / \mathrm{g})\end{array}$ & $\begin{array}{c}\text { Maximum } \\
(\mu \mathrm{g} / \mathrm{g})\end{array}$ \\
\hline \multirow[t]{5}{*}{ July } & Stems & 4.8 & 0.4 & 2.8 & 7.8 \\
\hline & Leaves & 4.4 & 0.5 & 1.0 & 8.4 \\
\hline & Shoots ${ }^{\mathrm{a}}$ & 4.7 & 0.2 & 2.8 & 6.4 \\
\hline & Rhizomes & 29.4 & 12.8 & 5.4 & 232.2 \\
\hline & Roots $^{b}$ & 101.4 & 23.6 & 21.5 & 316.0 \\
\hline \multirow[t]{4}{*}{ December } & Stems & 7.4 & 0.8 & 1.0 & 14.3 \\
\hline & Leaves $^{c}$ & 9.0 & 3.6 & 3.9 & 33.6 \\
\hline & Flowers/seeds $^{d}$ & 5.1 & 0.7 & 3.4 & 7.9 \\
\hline & Shoots $^{\mathrm{a}}$ & 7.3 & 0.8 & 0.9 & 13.3 \\
\hline \multicolumn{6}{|c|}{$\begin{array}{l}\text { alue for each sampling site was a weighted average (based on tissue mass) of concentration } \\
\text { data for stems and leaves (July) and stems, leaves, and seeds (December). } \\
\text { b } n=14 \\
{ }_{n} n=8 \\
{ }_{n}=6\end{array}$} \\
\hline
\end{tabular}

\begin{tabular}{|c|c|c|c|c|c|}
\hline Sample date & Plant part & Mean & SE & Minimum & Maximum \\
\hline \multirow[t]{3}{*}{ July } & Shoots & 0.015 & 0.004 & 0.004 & 0.056 \\
\hline & Rhizomes & 0.072 & 0.021 & 0.007 & 0.330 \\
\hline & Roots & 0.232 & 0.061 & 0.029 & 0.726 \\
\hline December & Shoots & 0.020 & 0.004 & 0.002 & 0.065 \\
\hline
\end{tabular}




\begin{tabular}{|c|c|c|c|c|c|}
\hline $\begin{array}{l}\text { Sample } \\
\text { location }\end{array}$ & Sample date & $\begin{array}{l}\text { Mean } \\
(\mu \mathrm{g})\end{array}$ & $\begin{array}{c}\text { SE } \\
(\mu \mathrm{g})\end{array}$ & $\begin{array}{c}\text { Minimum } \\
(\mu \mathrm{g})\end{array}$ & $\begin{array}{l}\text { Maximum } \\
(\mu \mathrm{g})\end{array}$ \\
\hline \multirow{2}{*}{$\begin{array}{l}\text { Upland sites } \\
\mathrm{n}=8\end{array}$} & July & 78 & 9 & 36 & 112 \\
\hline & December & 291 & 81 & 57 & 704 \\
\hline \multirow{2}{*}{$\begin{array}{l}\text { Marsh sites } \\
\mathrm{n}=9\end{array}$} & July & 313 & 36 & 150 & 492 \\
\hline & December & 828 & 118 & 491 & 1677 \\
\hline \multirow{2}{*}{$\begin{array}{r}\text { All sites } \\
\mathrm{n}=17\end{array}$} & July & 202 & 35 & 36 & 492 \\
\hline & December & 575 & 97 & 57 & 1677 \\
\hline
\end{tabular}

\begin{tabular}{|c|c|c|}
\hline Edaphic factor & July & December \\
\hline Total $\mathrm{Pb}(\mu \mathrm{g} / \mathrm{g})$ & 0.46 & 0.37 \\
\hline Available $\mathrm{Pb}(\mu \mathrm{g} / \mathrm{g})$ & $0.70^{* *}$ & $0.58^{*}$ \\
\hline $\mathrm{pH}$ & $-0.76^{* * *}$ & $-0.63 * *$ \\
\hline Organic matter (\%) & $0.77^{* * *}$ & $0.51^{*}$ \\
\hline Available $\mathrm{P}(\mu \mathrm{g} / \mathrm{g})$ & -0.07 & -0.16 \\
\hline Cation exchange capacity (meq) & $0.79 * * *$ & $0.50 *$ \\
\hline Electrical conductivity (dS/m) & $0.74 * * *$ & 0.47 \\
\hline
\end{tabular}


Table 11. Estimated biomass and $\mathrm{Pb}$ uptake for Phragmites shoots in December for sites where total $\mathrm{Pb}$ in soil is $<1000 \mu \mathrm{g} / \mathrm{g}$

\begin{tabular}{|l|l|c|c|c|c|}
\hline $\begin{array}{c}\text { Sample } \\
\text { location }\end{array}$ & \multicolumn{1}{|c|}{ Variable } & Mean & SE & Minimum & Maximum \\
\hline $\begin{array}{c}\text { Upland sites } \\
\mathrm{n}=8\end{array}$ & $\begin{array}{l}\text { Sampled area } \\
\text { biomass }(\mathrm{g} \text { dry wt) }\end{array}$ & 94 & 18 & 23 & 148 \\
\cline { 2 - 6 } & $\begin{array}{l}\text { Sampled area Pb } \\
\text { uptake }(\mu \mathrm{g})^{\mathrm{b}}\end{array}$ & 470 & 110 & 116 & 999 \\
\cline { 2 - 6 } & Pb uptake $\left(\mathrm{mg} / \mathrm{m}^{2}\right)^{\mathrm{c}}$ & 15 & 3 & 4 & 31 \\
\hline \multirow{2}{*}{$\begin{array}{c}\text { Marsh sites } \\
\mathrm{n}=9\end{array}$} & $\begin{array}{l}\text { Sampled area } \\
\text { biomass }(\mathrm{g} \text { dry wt) }\end{array}$ & 152 & 14 & 82 & 215 \\
\cline { 2 - 6 } & $\begin{array}{l}\text { Sampled area Pb } \\
\text { uptake }(\mu \mathrm{gg})^{\mathrm{b}}\end{array}$ & 1379 & 203 & 858 & 2879 \\
\cline { 2 - 6 } & Pb uptake $\left(\mathrm{mg} / \mathrm{m}^{2}\right)^{\mathrm{c}}$ & 43 & 6 & 26 & 89 \\
\hline
\end{tabular}

${ }^{a}$ The sum of the measured stem and flower/seed masses plus an estimated leaf mass based on the average relationship between leaf mass and stem mass for the marsh and upland areas in July.

${ }^{\mathrm{b}}$ Calculated using estimated leaf and flower/seed $\mathrm{Pb}$ concentrations for sites where leaf samples were not available or flower/seed samples were not analyzed. Estimated leaf and flower/seed $\mathrm{Pb}$ concentrations were calculated by using the average relationships between stem $\mathrm{Pb}$ concentrations and leaf or flower/seed $\mathrm{Pb}$ concentrations for the marsh and upland areas.

${ }^{c}$ Assumes that the plant mass in the sampled area $(18 \times 18 \mathrm{~cm})$ is representative of an average potential plant production per $\mathrm{m}^{2}$. 


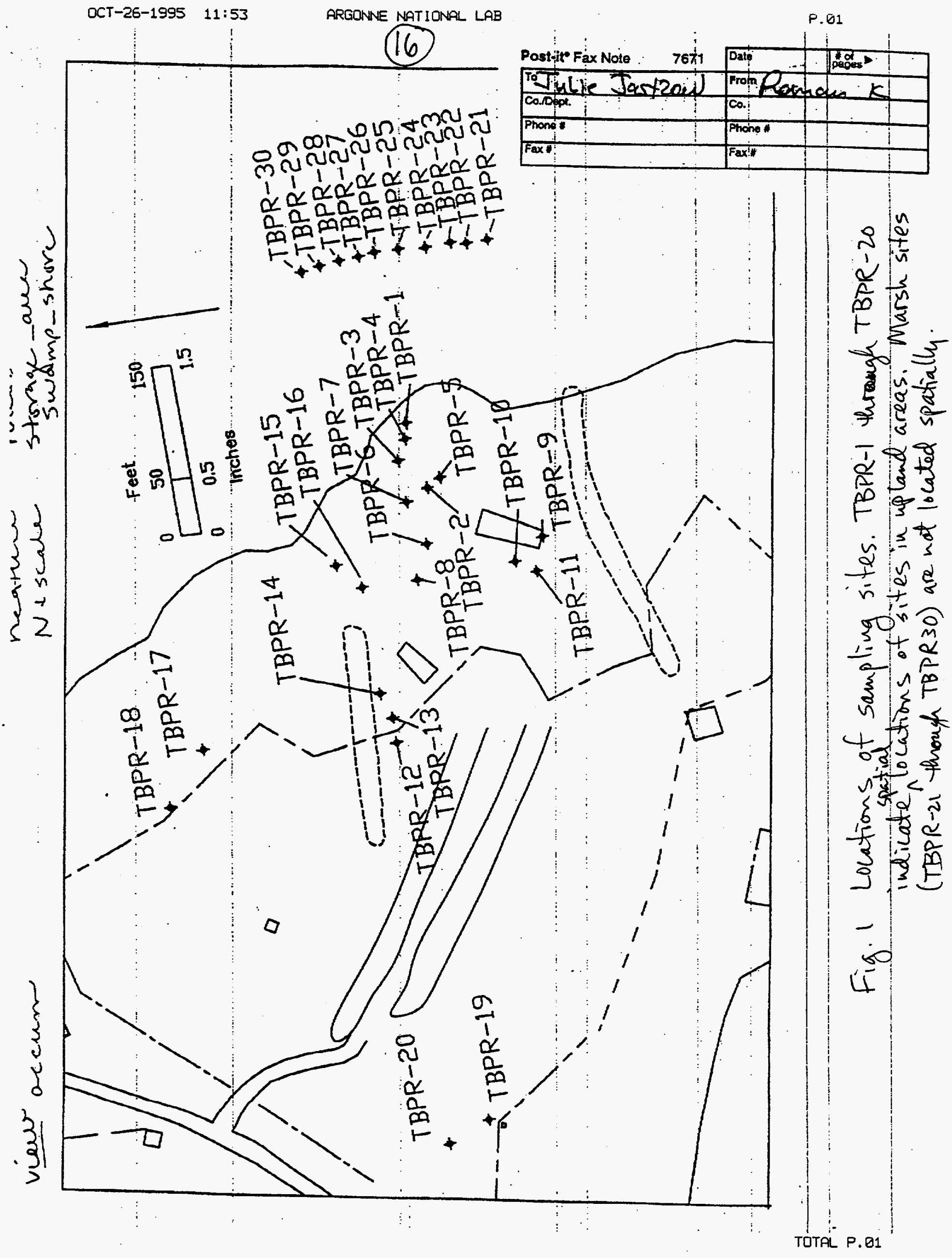


SA

17

16:02 Friday, November 3, 19952.

TIME $=2$

Plot of SHPB*AVPB. Symbol used is $1 * 1$.

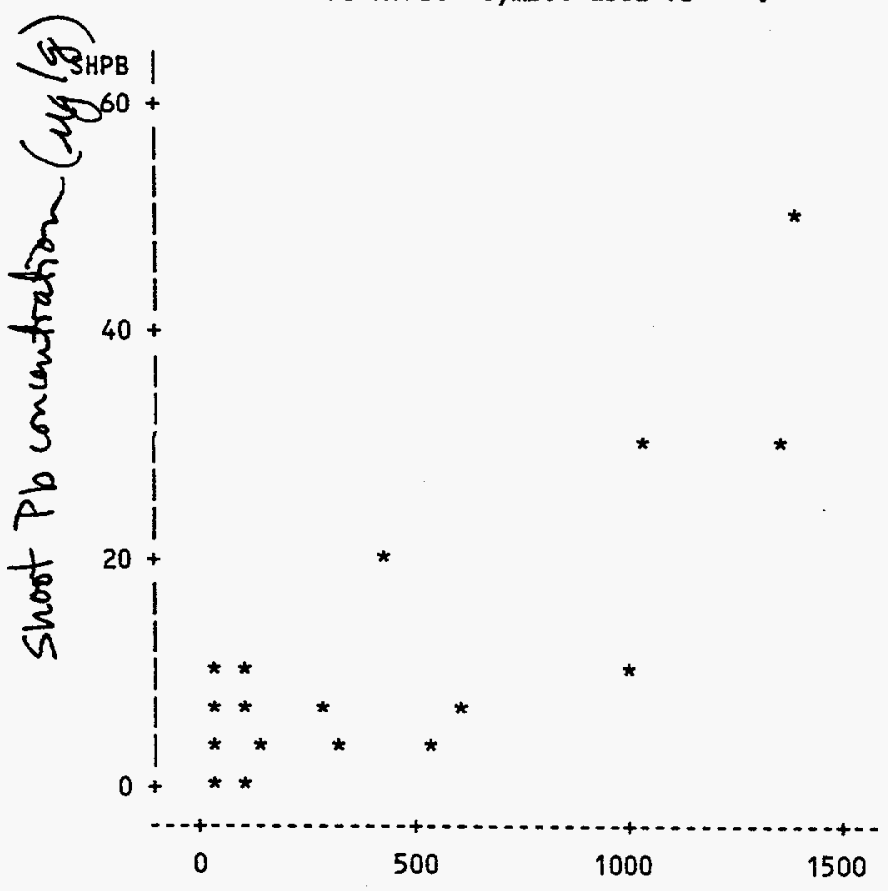

Available Pb in Soil

NOTE: 3 obs hidden.

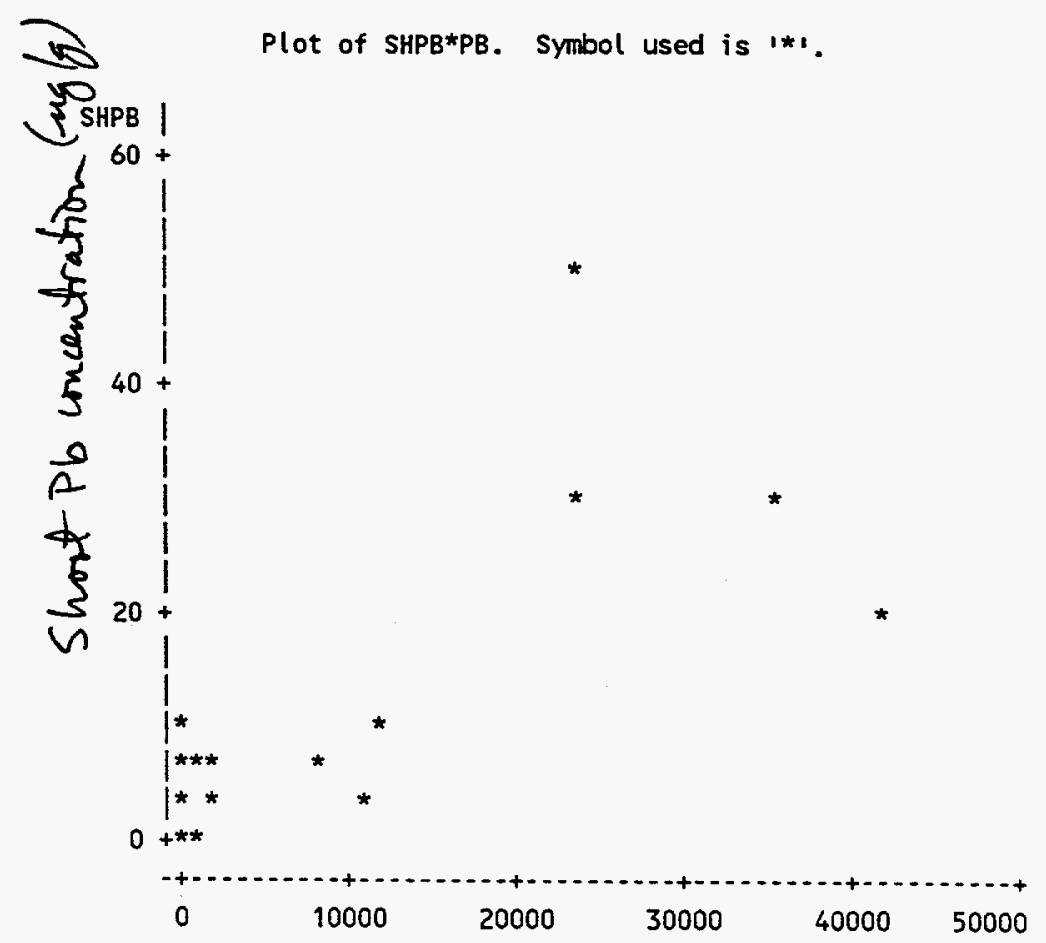

Total sol Pb

NOTE: 5 obs hidden.

Fig. 2 Relationships between Po $_{\text {tissue }}$ concentrations in Phragmites shots and available (DTPA-extraltabt and total $P b$ in soil in December for all 20 upland sampling sites. 
Plot of SHPB*AVPB. Symbol is value of LOC.

Plot of SHPB*PB. Symbol is value of LOC.
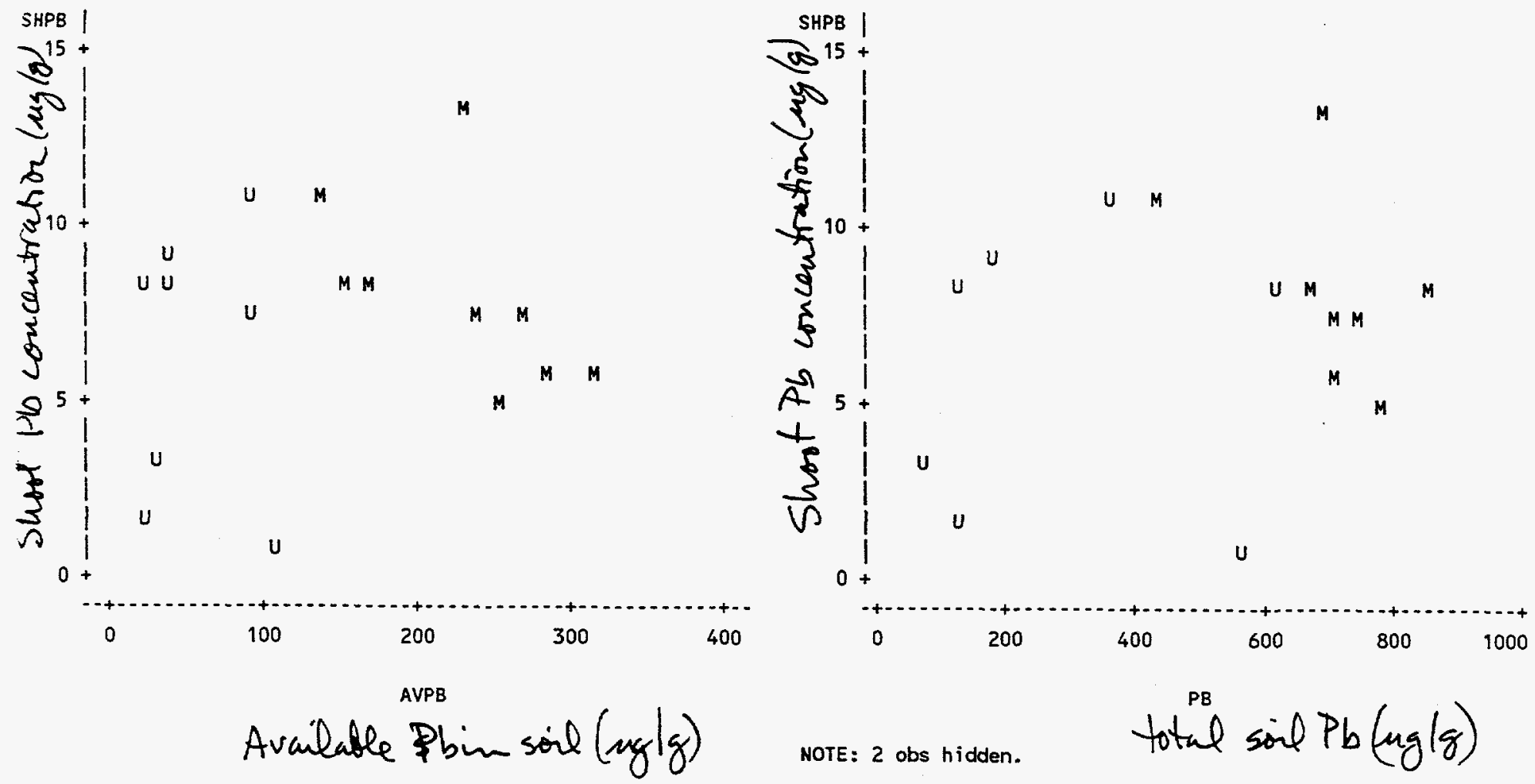

$$
\begin{aligned}
& \text { Fig. } 3 \text { Relationships between } \mathrm{Pb} \text { tissue concentrations in } \\
& \text { Phragnites shots and available (DTPA-extractable) } \\
& \text { and total Pb in soil in December for sites with } \\
& <1000 \mu g / g \text { total } \mathrm{Pb} \text { in thesoil. } U=\text { upland sites; } \\
& \\
& M=\text { marsh sites. }
\end{aligned}
$$

\section{DISCLAIMER}

This report was prepared as an account of work sponsored by an agency of the United States Government. Neither the United States Government nor any agency thereof, nor any of their employees, makes any warranty, express or implied, or assumes any legal liability or responsibility for the accuracy, completeness, or usefulness of any information, apparatus, product, or process disclosed, or represents that its use would not infringe privately owned rights. Reference herein to any specific commercial product, process, or service by trade name, trademark, manufacturer, or otherwise does not necessarily constitute or imply its endorsement, recommendation, or favoring by the United States Government or any agency thereof. The views and opinions of authors expressed herein do not necessarily state or reflect those of the United States Government or any agency thereof. 
APPENDIX 
Table A.l Results of soil analyses.

\begin{tabular}{|c|c|c|c|c|c|c|c|c|c|c|c|c|c|c|c|}
\hline & & & & \multicolumn{3}{|c|}{ AVAlL AVAlL } & EXCH & EXCH & & & & & & & \\
\hline SITEI & & & & $\mathrm{pH}$ & $\mathrm{P}$ & $\mathrm{K}$ & $\mathrm{Ca}$ & $\mathrm{Mg}$ & $\mathrm{N}$ & CEC & BSCA & BSMG & BSK & O.M. & $E C$ \\
\hline STUDY & ID & $\angle A B$ & TYPE & & $(\mathrm{mg} / \mathrm{kg})$ & $\mathrm{mg} / \mathrm{kg}$ & $(\mathrm{mg} / \mathrm{kg})$ & $(\mathrm{mg} / \mathrm{kg})$ & (\%) & (meq) & $(\%)$ & $(\%)$ & (\%) & $(\%)$ & $(\mathrm{dS} / \mathrm{cm})$ \\
\hline TBPR & 1 & OSU & & 6.3 & 106 & 108 & 855 & 333 & & 7 & 49 & 32 & 3.2 & 4.9 & 0.20 \\
\hline TBPR & 2 & OSU & & 6.5 & 2 & 51 & 302 & 76 & & 2 & 67 & 28 & 5.7 & 3.7 & 0.12 \\
\hline TBPR & 3 & OSU & & 6.4 & 7 & 67 & 243 & 78 & & 2 & 60 & 32 & 8.4 & 3.5 & 0.12 \\
\hline TBPR & 3 & WIS & DUP & & & & & & & & & & & & \\
\hline TBPR & 4 & OSU & & 6.1 & 1 & 38 & 196 & 68 & & 3 & 36 & 21 & 3.5 & 3.3 & 0.12 \\
\hline TBPR & 4 & WIS & & 5.8 & 4 & 50 & 250 & 60 & 0.08 & & & & & 3.4 & 0.06 \\
\hline TBPR & 4 & WIS & DUP & & & & & & & & & & & & \\
\hline TBPR & 5 & OSU & & 6.6 & 9 & 54 & 539 & 96 & & 4 & 74 & 22 & 3.8 & 3.7 & $<0.10$ \\
\hline TBPR & 5 & WIS & & 6.1 & 13 & 50 & 500 & 90 & 0.07 & & & & & 3.1 & 0.05 \\
\hline TBPR & 6 & OSU & & 7.2 & 49 & 80 & 970 & 385 & & 8 & 59 & 39 & 2.5 & 3.0 & 0.20 \\
\hline TBPR & 6 & OSU & DUP & 7.3 & 49 & 71 & 927 & 364 & & 8 & 59 & 38 & 2.7 & 3.2 & 0.17 \\
\hline TBPR & 7 & OSU & & 7.1 & 234 & 96 & 866 & 309 & & 6 & 61 & 36 & 3.4 & 3.8 & 0.11 \\
\hline TBPR & 7 & WIS & & 5.7 & 200 & 75 & 700 & 290 & 0.12 & & & & & 3.3 & 0.07 \\
\hline TBPR & 8 & OSU & & 8.4 & 128 & 75 & 1047 & 971 & & 12 & 39 & 60 & 1.4 & 2.2 & 1.08 \\
\hline TBPR & 8 & WIS & DUP & & & & & & & & & & & & \\
\hline TBPR & 9 & OSU & & 7.4 & 55 & 49 & 948 & 118 & & 6 & 81 & 17 & 2.1 & 2.0 & 0.43 \\
\hline TBPR & 10 & OSU & & 7.6 & 45 & 92 & 1125 & 259 & & 7 & 70 & 27 & 2.9 & 2.5 & 0.22 \\
\hline TBPR & 10 & WIS & DUP & & & & & & & & & & & & \\
\hline TBPR & 11 & Osu & & 7.4 & 35 & 23 & 1278 & 86 & & 7 & 89 & 10 & 0.8 & 1.0 & 1.08 \\
\hline TBPR & 11 & WIS & & 6.7 & 33 & 40 & 1250 & 100 & 0.03 & & & & & 0.9 & 0.69 \\
\hline TBPR & 11 & WIS & DUP & & & & & & & & & & & & \\
\hline TBPR & 12 & OSU & & 8.6 & 188 & 52 & 663 & 854 & & 11 & 31 & 67 & 1.3 & 1.8 & 0.19 \\
\hline TBPR & 12 & OSU & DUP & 8.7 & 192 & 54 & 651 & 789 & & 10 & 33 & 66 & 1.4 & 1.6 & 0.19 \\
\hline TBPR & 13 & OSU & & 8.4 & 884 & 99 & 491 & 1268 & & 13 & 18 & 80 & 1.9 & 2.3 & 0.26 \\
\hline TBPR & 13 & WIS & DUP & & & & & & & & & & & & \\
\hline TBPR & 14 & OSU & & 8.6 & 61 & 53 & 742 & 565 & & 9 & 43 & 55 & 1.6 & 1.6 & 0.28 \\
\hline TBPR & 14 & WIS & & 6.9 & 56 & 50 & 750 & 440 & 0.04 & & & & & 1.5 & 0.23 \\
\hline TBPR & 15 & OSU & & 8.0 & 15 & 17 & 415 & 174 & & 4 & 58 & 41 & 1.2 & 0.8 & 0.11 \\
\hline TBPR & 15 & OSU & DUP & 8.0 & 11 & 19 & 420 & 169 & & 4 & 59 & 40 & 1.4 & 0.7 & $<0.10$ \\
\hline TBPR & 16 & OSU & & 8.0 & 130 & 77 & 907 & 781 & & 9 & 40 & 58 & 1.7 & 2.5 & 0.36 \\
\hline TBPR & 17 & OSU & & 8.6 & 57 & 53 & 619 & 929 & & 10 & 28 & 71 & 1.2 & 2.2 & 0.38 \\
\hline TBPR & 17 & WIS & DUP & & & & & & & & & & & & \\
\hline TBPR & 18 & osu & & 7.3 & 17 & 26 & 563 & 120 & & 4 & 72 & 26 & 1.7 & 1.1 & $<0.10$ \\
\hline TBPR & 19 & OSU & & 6.2 & 9 & 46 & 592 & 92 & & 3 & 77 & 20 & 3.1 & 2.0 & $<0.10$ \\
\hline TBPR & 19 & WIS & & 6.6 & 8 & 50 & 500 & 100 & 0.06 & & & & & 1.5 & 0.04 \\
\hline TBPR & 20 & OSU & & 6.5 & 10 & 39 & 585 & 92 & & 4 & 77 & 20 & 2.6 & 1.6 & $<0.10$ \\
\hline TBPR & 21 & OSU & & 5.7 & 60 & 206 & 1100 & 1043 & & 15 & 23 & 36 & 2.2 & 7.4 & 1.90 \\
\hline TBPA & 21 & WIS & DUP & & & & & & & & & & & & \\
\hline TBPR & 22 & OSU & & 6.0 & 55 & 201 & 1371 & 1265 & & 15 & 29 & 45 & 2.2 & 7.9 & 1.50 \\
\hline TBPR & 23 & osu & & 5.7 & 87 & 323 & 1316 & 1241 & & 16 & 26 & 41 & 3.3 & 9.0 & 2.60 \\
\hline TBPR & 24 & OSU & & 5.7 & 113 & 289 & 1732 & 1525 & & 20 & 25 & 36 & 2.1 & 14.1 & 3.45 \\
\hline TBPR & 25 & osu & & 5.6 & 100 & 275 & 1618 & 1528 & & 18 & 25 & 40 & 2.2 & 10.6 & 2.70 \\
\hline TBPR & 26 & IOSU & & 5.6 & 104 & 348 & 1667 & 1470 & & 21 & 23 & 34 & 2.5 & 12.8 & 1.88 \\
\hline TBPR & 27 & OSU & & 5.3 & 185 & 571 & 2463 & 1885 & & 20 & 25 & 31 & 2.9 & 20.7 & 4.00 \\
\hline TBPR & 28 & OSU & & 5.6 & 92 & 387 & 1965 & 1467 & & 20 & 27 & 34 & 2.7 & 17.5 & 5.50 \\
\hline TBPR & 29 & OSU & & 5.3 & 204 & 412 & 4187 & 2336 & & 29 & 36 & 33 & 1.8 & 22.5 & 8.45 \\
\hline TBPR & 30 & OSU & & 6.8 & 49 & 96 & 2310 & 1147 & & 15 & 54 & 45 & 1.2 & 8.9 & 2.54 \\
\hline
\end{tabular}


Table A.I cont.

\begin{tabular}{|c|c|c|c|c|c|c|c|c|c|c|c|c|c|c|c|}
\hline SITE & & & & $\mathrm{Pb}$ & $\mathrm{Cd}$ & $\mathrm{Ni}$ & $\mathrm{Cr}$ & $\mathrm{Cu}$ & $\mathrm{Zn}$ & $\mathrm{Hg}$ & As & Mo & Se & $\mathrm{Fe}$ & Co \\
\hline STUDY & ID & LAB & TYPE & $(\mathrm{mg} / \mathrm{kg})$ & $(\mathrm{mg} / \mathrm{kg})$ & $(\mathrm{mg} / \mathrm{kg})$ & $(\mathrm{mg} / \mathrm{kg})$ & $(\mathrm{mg} / \mathrm{kg})$ & $(\mathrm{mg} / \mathrm{kg})$ & (mg/kg) & (mg/kg) & $(\mathrm{mg} / \mathrm{kg})$ & (mg/kg) & $(\mathrm{mg} / \mathrm{kg})$ & $(\mathrm{mg} / \mathrm{kg})$ \\
\hline TBPR & 1 & OSU & & 11737 & 1.09 & 42.8 & 175.3 & 780 & 2900 & $<1.0$ & 22.2 & $<0.2$ & 0.06 & & \\
\hline TBPA & 2 & OSU & & 41414 & 5.96 & 46.0 & 242.5 & 2692 & 9079 & $<1.0$ & 27.6 & $<0.2$ & 0.05 & & \\
\hline TBPR & 3 & OSU & & 35704 & 1.75 & 50.0 & 166.7 & 821 & 4966 & $<1.0$ & 34.9 & $<0.2$ & 0.06 & & \\
\hline TBPR & 3 & WIS & DUP & & & & & & & & & & & & \\
\hline TBPR & 4 & OSU & & 25053 & 0.87 & 32.8 & 159.3 & 918 & 2686 & $<1.9$ & 23.7 & $<0.2$ & 0.05 & & \\
\hline TBPR & 4 & WIS & & 23316 & 7.14 & 27.9 & 141.5 & 807 & 2330 & & $<27.9$ & & & 78353 & 5.31 \\
\hline TBPR & 4 & WIS & DUP & 21661 & 13.25 & 28.2 & 397.1 & 888 & 2367 & & $<27.9$ & & & 72483 & 4.53 \\
\hline TBPR & 5 & OSU & & 23108 & 2.52 & 58.4 & 316.5 & 1699 & 7963 & $<1.0$ & 34.5 & $<0.2$ & 0.04 & & \\
\hline TBPR & 5 & WIS & & 23464 & 10.71 & 40.8 & 313.4 & 1328 & 6186 & & $<27.9$ & & & 66671 & 10.52 \\
\hline TBPR & 6 & OSU & & 9553 & 3.92 & 39.0 & 168.0 & 1316 & 4689 & $<1.0$ & 24.7 & $<0.2$ & 0.05 & & \\
\hline TBPR & 6 & osu & DUP & 6696 & 3.62 & 37.7 & 151.9 & 1054 & 4919 & $<1.0$ & 26.3 & $<0.2$ & 0.08 & & \\
\hline TBPA & 7 & OSU & & 10607 & 5.85 & 39.9 & 254.5 & 1001 & 5569 & $<1.0$ & 26.5 & $<0.2$ & 0.07 & & \\
\hline TBPA & 7 & WIS & & 10368 & 6.42 & 22.9 & 150.9 & 593 & 4259 & & $<27.9$ & & & 46115 & 5.39 \\
\hline TBPR & 8 & OSU & & 1950 & 5.15 & 22.4 & 127.6 & 524 & 1284 & $\leq 1.9$ & 22.0 & $<0.2$ & 0.14 & & \\
\hline TBPR & 8 & WIS & DUP & & & & & & & & & & & & \\
\hline TBPR & 9 & osu & & 1447 & 0.81 & 19.9 & 152.3 & 691 & 894 & $<1.0$ & 17.3 & $<0.2$ & 0.11 & & \\
\hline TBPR & 10 & OSU & & 1746 & 1.63 & 25.7 & 105.3 & 388 & 1645 & $<1.0$ & 47.8 & $<0.2$ & 0.10 & & \\
\hline TBPR & 10 & WIS & DUP & & & & & & & & & & & & \\
\hline TBPR & 11 & OSU & & 3211 & 1.07 & 16.7 & 53.0 & 204 & 1001 & $<1.9$ & 17.9 & $<0.2$ & 0.09 & & \\
\hline TBPA & 11 & WIS & & 423 & 3.99 & 15.5 & 229.8 & 377 & 783 & & $<27.9$ & & & 18144 & 3.13 \\
\hline TBPR & 11 & WIS & DUP & 1596 & 4.02 & 15.5 & 106.7 & 433 & 879 & & $<27.9$ & & & 23457 & 3.16 \\
\hline TBPR & 12 & OSU & & 393 & 3.80 & 25.3 & 89.5 & 506 & 1906 & $<1.0$ & 12.6 & $<0.2$ & 0.08 & & \\
\hline TBPR & 12 & Osu & DUP & 351 & 5.08 & 22.4 & 48.7 & 360 & 1785 & $<1.0$ & 12.0 & $<0.2$ & 0.11 & & \\
\hline TBPR & 13 & OSU & & 610 & 3.65 & 45.9 & 148.8 & 496 & 1552 & $<1.0$ & 18.6 & 0.6 & 0.10 & & \\
\hline TBPR & 13 & WIS & DUP & & & & & & & & & & & & \\
\hline TBPR & 14 & OSU & & 496 & 2.18 & 22.1 & 82.2 & 361 & 936 & $<1.0$ & 12.2 & $<0.2$ & 0.08 & & \\
\hline TBPR & 14 & WIS & & 995 & 1.79 & 9.1 & 109.9 & 189 & 872 & & $<27.9$ & & & 12827 & 3.36 \\
\hline TBPR & 15 & IOSU & & 60 & 0.62 & 9.2 & 28.4 & 84 & 132 & $<1.0$ & 3.9 & $<0.2$ & 0.07 & & \\
\hline TBPR & 15 & osu & DUP & 189 & 0.45 & 8.4 & 29.8 & 65 & 123 & $<1.0$ & 4.1 & $<0.2$ & 0.10 & & \\
\hline TBPR & 16 & OSU & & 1205 & 1.78 & 27.8 & 96.0 & 348 & 988 & $<1.0$ & 16.8 & $<0.2$ & 0.19 & & \\
\hline TBPR & 17 & OSU & & $564:$ & 1.32 & 25.6 & 48.6 & 330 & 914 & $<1.0$ & 11.9 & $<0.2$ & 0.15 & & \\
\hline TBPR & 17 & WIS & DUP & & & & & & & & & & & & \\
\hline TBPR & 18 & OSU & & 121 & 0.95 & 11.5 & 30.3 & 101 & 201 & $<1.0$ & 5.9 & $<0.2$ & 0.07 & & \\
\hline TBPR & 19 & OSU & & 79 & $<0.20$ & 11.6 & 52.0 & 255 & 209 & $<1.0$ & 4.7 & $<0.2$ & 0.08 & & \\
\hline TBPR & 19 & WIS & & 84 & $<1.00$ & 6.9 & 70.4 & 203 & 170 & & $<27.9$ & & & 11039 & 3.17 \\
\hline TBPR & 20 & osu & & 173 & 1.36 & 11.7 & 48.1 & 232 & 272 & $<1.0$ & 5.3 & $<0.2$ & 0.08 & & \\
\hline TBPR & 21 & OSU & & 746 & $<0.20$ & 43.0 & 95.6 & 401 & 1076 & $<1.0$ & 41.9 & 0.8 & 0.15 & & \\
\hline TBPR & 21 & WIS & DUP & & & & & & & & & &. & & \\
\hline TBPR & 22 & OSU & & 784 & $<0.20$ & 51.4 & 117.8 & 507 & 1413 & $<1.0$ & 43.6 & $<0.2$ & 0.15 & & \\
\hline TBPR & 23 & OSU & & 704 & 0.44 & 46.5 & 94.7 & 452 & 1302 & $<1.0$ & 42.5 & $<0.2$ & 0.17 & & \\
\hline TBPR & 24 & OSU & & 685 & 0.80 & 37.3 & 91.4 & 426 & 983 & $<1.0$ & 39.3 & 1.0 & 0.12 & & \\
\hline TBPR & 25 & OSU & & 711 & $<0.20$ & 48.3 & 104.0 & 445 & 1194 & $<1.0$ & 40.3 & $<0.2$ & 0.22 & & \\
\hline TBPR & 26 & osu & & 703 & $<0.20$ & 39.9 & 89.3 & 312 & 947 & $<1.0$ & 25.7 & $<0.2$ & 0.28 & & \\
\hline TBPR & 27 & OSU & & 665 & 0.45 & 29.7 & 79.0 & 322 & 747 & $<1.0$ & 25.6 & 1.1 & 0.29 & & \\
\hline TBPR & 28 & IOSU & & 848 & 1.21 & 30.3 & 63.6 & 393 & 1005 & $<1.0$ & 27.5 & $<0.2$ & 0.28 & & \\
\hline TBPR & 29 & OSU & & 445 & 2.01 & 21.1 & 69.4 & 346 & 952 & $<1.0$ & 18.6 & $<0.2$ & 0.25 & & \\
\hline TBPR & 30 & OSU & & 1258 & 0.87 & 24.1 & 78.6 & 387 & 1417 & $<1.0$ & 17.9 & $<0.2$ & 0.09 & & \\
\hline
\end{tabular}




\begin{tabular}{|c|c|c|c|c|c|c|c|c|c|c|c|}
\hline & & & & DTPA & DTPA & DTPA & DTPA & DTPA & DTPA & DTPA & DTPA \\
\hline SITE & & & & $\mathrm{Cd}$ & $\mathrm{Cr}$ & $\mathrm{Cu}$ & $\mathrm{Zn}$ & $\mathrm{Ni}$ & $\mathrm{Pb}$ & $\mathrm{Fe}$ & $\mathrm{Mn}$ \\
\hline STUDY & ID & LAB & TYPE & $(\mathrm{mg} / \mathrm{kg})$ & $(\mathrm{mg} / \mathrm{kg})$ & $(\mathrm{mg} / \mathrm{kg})$ & $(\mathrm{mg} / \mathrm{kg})$ & $(\mathrm{mg} / \mathrm{kg})$ & $(\mathrm{mg} / \mathrm{kg})$ & $(\mathrm{mg} / \mathrm{kg})$ & $(\mathrm{mg} / \mathrm{kg})$ \\
\hline TBPR & 1 & WIS & & $\begin{array}{r}1.42 \\
\end{array}$ & 0.41 & 212.8 & 762.7 & 2.22 & 998.1 & 86.18 & 6.13 \\
\hline TBPR & 2 & WIS & & 1.48 & $<0.08$ & 224.9 & 1083.0 & 1.28 & 424.8 & 1.69 & 0.51 \\
\hline TBPR & 3 & WIS & & 1.50 & 0.14 & 136.7 & 663.9 & 1.72 & 1324.0 & 9.34 & 1.53 \\
\hline TBPR & 3 & WIS & DUP & 1.38 & 0.09 & 125.4 & 594.4 & 1.46 & 1385.0 & 9.76 & 1.53 \\
\hline TBPR & 4 & WIS & & 0.49 & $<0.08$ & 158.3 & 391.3 & 0.75 & 1408.0 & 49.91 & 1.40 \\
\hline TBPR & 4 & WIS & & & & & & & & & \\
\hline TBPR & 4 & WIS & DUP & & & & & & & & \\
\hline TBPR & 5 & WIS & & 1.39 & 1.14 & 230.8 & 745.7 & 0.93 & 1028.0 & 15.42 & 1.35 \\
\hline TBPR & 5 & WIS & & & & & & & & & \\
\hline TBPR & 6 & WIS & & 1.87 & 0.30 & 203.3 & 608.4 & 0.64 & 617.9 & 49.06 & 8.13 \\
\hline TBPR & 6 & WIS & DUP & & & & & & & & \\
\hline TBPR & 7 & WIS & & 1.85 & 0.09 & 145.1 & 956.8 & 1.44 & 531.4 & 85.25 & 4.98 \\
\hline TBPR & 7 & WIS & & & & & & & & & \\
\hline TBPR & 8 & WIS & & 2.18 & 0.48 & 114.8 & 145.3 & 0.31 & 279.8 & 57.99 & 6.61 \\
\hline TBPR & 8 & WIS & DUP & 1.80 & 0.40 & 99.5 & 130.9 & 0.26 & 279.4 & 53.53 & 5.71 \\
\hline TBPR & 9 & WIS & & 0.73 & 0.16 & 116.2 & 97.7 & $<0.18$ & 129.2 & 19.08 & 8.42 \\
\hline TBPR & 10 & WIS & & 1.18 & 0.17 & 76.8 & 200.2 & 0.27 & 340.0 & 49.05 & 7.94 \\
\hline TBPR & 10 & WIS & DUP & 1.13 & $<0.08$ & 77.1 & 186.7 & 0.21 & 332.5 & 47.98 & 7.88 \\
\hline TBPR & 11 & WIS & & 0.69 & 0.15 & 45.7 & 171.4 & $<0.18$ & 312.6 & 19.00 & 7.67 \\
\hline TBPR & 11 & WIS & & & & & & & & & \\
\hline TBPR & 11 & WIS & DUP & & & & & & & & \\
\hline TBPR & 12 & WIS & & 1.27 & 0.22 & 54.2 & 273.9 & 0.35 & 91.2 & 32.98 & 3.51 \\
\hline TBPR & 12 & WIS & DUP & & & & & & & & \\
\hline TBPR & 13 & WIS & & 0.91 & 0.18 & 44.6 & 207.3 & 0.62 & 35.9 & 82.93 & 5.60 \\
\hline TBPR & 13 & WIS & DUP & 0.85 & $<0.08$ & 42.7 & 184.7 & 0.53 & 35.7 & 79.75 & 5.29 \\
\hline TBPR & 14 & WIS & & 0.87 & 0.12 & 64.8 & 115.4 & 0.24 & 90.4 & 49.91 & 5.08 \\
\hline TBPR & 14 & WIS & & & & & & & & & \\
\hline TBPR & 15 & WIS & & 0.54 & 0.14 & 17.1 & 9.6 & $<0.18$ & 19.7 & 13.18 & 4.61 \\
\hline TBPR & 15 & WIS & DUP & & & & & & & & \\
\hline TBPR & 16 & WIS & & 1.27 & 0.15 & 73.1 & 107.9 & 0.33 & 111.8 & 71.86 & 7.54 \\
\hline TBPR & 17 & WIS & & 1.11 & 0.19 & 58.4 & 96.3 & 0.36 & 102.3 & 49.50 & 7.33 \\
\hline TBPR & 17 & WIS & DUP & 1.11 & 0.16 & 56.0 & 99.8 & 0.38 & 113.1 & 50.59 & 7.38 \\
\hline TBPR & 18 & WIS & & 0.92 & $<0.08$ & 26.1 & 17.4 & $<0.18$ & 24.8 & 27.51 & 6.03 \\
\hline TBPR & 19 & WIS & & 0.59 & 0.21 & 91.0 & 37.8 & 0.31 & 29.8 & 45.48 & 9.39 \\
\hline TBPR & 19 & WIS & & & & & & & & & \\
\hline TBPR & 20 & WIS & & 2.19 & 0.18 & 105.0 & 48.9 & 0.28 & 40.9 & 29.27 & 11.75 \\
\hline TBPR & 21 & WIS & & 1.36 & 0.18 & 114.7 & 121.6 & 1.06 & 239.5 & 607.80 & 6.73 \\
\hline TBPR & 21 & WIS & DUP & 1.45 & 0.22 & 116.2 & 122.2 & 1.08 & 241.5 & 624.30 & 6.94 \\
\hline TBPR & 22 & WIS & & 1.80 & 0.16 & 134.2 & 154.7 & 1.27 & 254.5 & 442.20 & 8.43 \\
\hline TBPR & 23 & WIS & & 2.84 & 0.17 & 145.2 & 206.7 & 1.30 & 269.3 & 551.90 & 7.82 \\
\hline TBPR & 24 & WIS & & 2.63 & 0.21 & 157.3 & 198.4 & 1.87 & 230.0 & 774.40 & 9.34 \\
\hline TBPR & 25 & WIS & & 1.65 & 0.21 & 144.2 & 172.8 & 1.50 & 286.7 & 569.80 & 7.83 \\
\hline TBPR & 26 & WIS & & 1.62 & 0.19 & 112.2 & 156.0 & 1.71 & 318.8 & 694.80 & 5.17 \\
\hline TBPR & 27 & WIS & & 1.09 & 0.28 & 56.9 & 107.9 & 1.95 & 153.3 & 891.90 & 9.90 \\
\hline TBPR & 28 & WIS & & 1.55 & 0.22 & 75.4 & 206.5 & 2.16 & 168.9 & 845.50 & 7.97 \\
\hline TBPR & 29 & WIS & & 2.40 & 0.24 & 138.6 & 325.2 & 2.35 & 140.4 & 666.70 & 9.07 \\
\hline TBPR & 30 & WIS & & 1.42 & 0.14 & 150.6 & 312.1 & 1.60 & 267.8 & 221.40 & 13.50 \\
\hline
\end{tabular}


Table A.2 Kesults of plant tissue analyses for July samples

\begin{tabular}{|c|c|c|c|c|c|c|c|c|c|c|c|c|c|c|c|c|c|c|c|c|c|c|}
\hline & & & & STEM & STEM & STEM & STEM & STEM & STEM & STEM & STEM & STEM & STEM & STEM & STEM & STEM & STEM & STEM & STEM & STEM & STEM & STEM \\
\hline SITEI & & & & $P$ & $K$ & $\mathrm{Ca}$ & $\mathrm{Mg}$ & $N$ & $\mathrm{Na}$ & $\mathrm{Fe}$ & $\overline{M n}$ & B & $\mathrm{Pb}$ & $\mathrm{Cd}$ & $\mathrm{Ni}$ & $\mathrm{Cr}$ & $\mathrm{Cu}$ & $\mathrm{Zn}$ & $\mathrm{Hg}$ & As & Mo & \\
\hline STUDY & ID & $\angle A B$ & TYYPE & (\%) & (\%) & $(\%)$ & (\%) & (\%) & $(u g / g)$ & $(\mathrm{ug} / \mathrm{g})$ & $(u g / g)$ & $(\mathrm{ug} / \mathrm{g})$ & $(\mathrm{ug} / \mathrm{g})$ & $(\mathrm{ug} / \mathrm{g})$ & $(\mathrm{ug} / \mathrm{g})$ & $(\mathrm{ug} / \mathrm{g})$ & $(u g / g)$ & $(\mathrm{ug} / \mathrm{g})$ & $(u g / g)$ & $(u g / g)$ & $(u g / g)$ & $(u g / g)$ \\
\hline TBPR & 1 & OSU & & 0.08 & 0.75 & 0.05 & 0.06 & 0.60 & 429.0 & 38.8 & 21.9 & 2.8 & 7.1 & $<0.2$ & 3.8 & $\begin{array}{r}1.5 \\
\end{array}$ & 6.5 & 83.4 & $-<1.9$ & $<20$ & 0.43 & $<0.02$ \\
\hline TBPR & 1 & WIS & & 0.07 & 0.77 & 0.05 & 0.06 & 0.45 & 420.0 & 15.6 & 19.7 & 3.6 & 7.5 & $<0.2$ & 2.3 & 0.8 & 5.9 & 75.3 & & $<2.0$ & & \\
\hline TBPA & 2 & OSU & & 0.03 & 0.45 & 0.03 & 0.05 & 0.42 & 133.1 & 34.4 & 2.5 & 2.4 & 14.8 & $<0.2$ & 2.4 & 1.8 & 6.1 & 117.2 & $<1.0$ & $<2.0$ & 0.38 & $<0.02$ \\
\hline TBPA & 3 & OSU & & 0.06 & 1.03 & 0.07 & 0.12 & 0.97 & 1432.4 & 44.7 & 5.8 & 2.9 & 22.9 & $<0.2$ & 2.7 & 2.0 & 8.3 & 441.6 & $<1.0$ & $<2.0$ & 0.40 & 0.02 \\
\hline TBPA & 3 & OSU & DUP & & & & & & & & & & & & & & & & & & & \\
\hline TBPA & 3 & WIS & & 0.07 & 1.12 & $\overline{0.07}$ & 0.12 & 0.85 & 1337.0 & 38.6 & 5.3 & 2.2 & 20.6 & $<0.2$ & 2.0 & 1.1 & 8.7 & 481.4 & & $<2.0$ & & \\
\hline TBPA & 4 & OSU & & 0.08 & 1.13 & 0.06 & 0.10 & 0.68 & 720.1 & 26.7 & 9.0 & 2.5 & 9.1 & $<0.2$ & 2.0 & 1.5 & 4.7 & 166.4 & $<1.0$ & $<2.0$ & 0.20 & $<0.02$ \\
\hline TBPA & 4 & OSU & DUP & & & & & & & & & & & & & & & & & & & \\
\hline TBPA & 4 & WIS & & & & & & & & & & & & & & & & & & & & \\
\hline TBPA & 4 & WIS & DUP & & & & & & & & & & & & & & & & & & & \\
\hline TBPA & 5 & OSU & & 0.03 & 0.81 & 0.11 & 0.10 & 0.57 & 245.5 & 59.1 & 3.7 & 3.7 & 19.5 & $<0.2$ & 3.4 & 2.7 & 10.0 & 287.2 & $<1.0$ & $<2.0$ & 0.56 & 0.03 \\
\hline TBPA & 6 & OSU & & 0.08 & 1.17 & 0.08 & 0.15 & 0.72 & 397.8 & 76.9 & 12.9 & 4.1 & 15.9 & $<0.2$ & 4.2 & 2.9 & 9.5 & 113.2 & $<1.0$ & $<2.0$ & 0.50 & $<0.02$ \\
\hline TBPR & 6 & OSU & DUP & 0.08 & 1.16 & 0.07 & 0.14 & 0.72 & 369.5 & 46.4 & 10.5 & 2.9 & 12.2 & $<0.2$ & 3.6 & 1.6 & 8.3 & 100.9 & $<1.0$ & $<2.0$ & 0.56 & 0.11 \\
\hline TBPA & 7 & OSU & & 0.07 & 0.51 & 0.04 & 0.07 & 0.60 & 310.3 & 46.9 & 12.1 & 3.3 & 7.3 & $\leq 0.2$ & 4.2 & 1.8 & 6.1 & 94.3 & $<1,0$ & $\leq 2.0$ & 0.43 & 0.02 \\
\hline TBPA & 8 & OSU & & 0.08 & 1.10 & 0.12 & 0.38 & 0.76 & 6917 & 40.0 & 20.1 & 5.1 & 7.7 & $<0.2$ & 2.9 & 2.3 & 15.7 & 147.0 & $\leq 1.0$ & $<2.0$ & 3.80 & 0.05 \\
\hline TBPR & 9 & OSU & & 0.12 & 1.38 & 0.22 & 0.09 & 0.95 & 270.4 & 64.9 & 70.1 & 4.5 & 8.3 & $<0.2$ & 4.9 & 3.5 & 18.2 & 173.8 & $<1.0$ & $<2.0$ & 0.33 & $<0.02$ \\
\hline TBPR & 9 & OSU & DUP & & & & & & & & & & & & & & & & & & & \\
\hline TBPR & 9 & WIS & & 0.09 & 1.56 & 0.20 & 0.10 & 0.81 & 285.0 & 52.5 & 65.0 & 2.5 & 6.0 & $<0.2$ & 2.2 & 1.6 & 16.9 & 139.1 & & $<2.0$ & & \\
\hline TBPR & 9 & WIS & DUP & & & & & & & 49.5 & 63.8 & & 6.2 & $<0.2$ & 2.1 & 1.6 & 17.0 & 138.5 & & $<2.0$ & & \\
\hline TBPR & 10 & OSU & & 0.10 & 0.92 & 0.12 & 0.09 & 0.53 & 198.1 & 43.8 & 23.6 & 3.7 & 8.4 & $<0.2$ & 6.1 & 2.2 & 14.8 & 159.4 & $<1.0$ & $<2.0$ & 0.54 & $<0.02$ \\
\hline TBPR & 10 & osu & DUP & 0.10 & 0.88 & 0.10 & 0.08 & 0.57 & 173.8 & 50.9 & 20.4 & 1.8 & 6.9 & $<0.2$ & 4.3 & 1.2 & 13.7 & 141.0 & $<10$ & $<2,0$ & 0.71 & 0.07 \\
\hline TBPR & 11 & IOSU & & 0.08 & 0.72 & 0.38 & 0.05 & 0.54 & 156.9 & 33.4 & 49.1 & 2.2 & 6.0 & $<0.2$ & 4.8 & 1.9 & 14.7 & 112.5 & $<1.0$ & $<2.0$ & 0.26 & 0.03 \\
\hline TBPR & 11 & TOSU & DUP & 0.08 & 0.69 & 0.33 & 0.05 & 0.48 & 132.2 & 34.9 & 45.5 & 1.3 & 6.2 & $<0.2$ & 4.6 & 0.9 & 13.7 & 102.5 & $<1.0$ & $<2.0$ & 0.43 & 0.06 \\
\hline TBPA & 12 & OSu & & 0.09 & 1.05 & 0.06 & 0.18 & 0.56 & 246.4 & 336 & 9.0 & 4.2 & 3.3 & $\leq 0.2$ & 2.8 & 2.5 & 9.9 & 84.0 & $<1.0$ & $<2.0$ & 0.43 & 0.07 \\
\hline TBPR & 13 & OSU & & 0.14 & 0.88 & 0.04 & 0.12 & 0.41 & 188.8 & 31.3 & 5.5 & 2.6 & 3.0 & $<0.2$ & 3.1 & 1.8 & 72 & 49.6 & $\leqslant 1.9$ & $<2.0$ & 0.58 & 0.07 \\
\hline TBPA & 14 & OSU & & 0.12 & 1.09 & 0.03 & 0.10 & 0.54 & 322.5 & 41.3 & 6.6. & 3.2 & 5.3 & $<0.2$ & 2.7 & 2.2 & 8.6 & 34.0 & $<1.0$ & $<2.0$ & 0.59 & 0.03 \\
\hline TBPR & 14 & WIS & & & & & & & & & & & & & & & & & & & & \\
\hline TBPA & 15 & OSU & & 0.11 & 1.10 & 0.10 & 0.08 & 0.60 & 221.6 & 36.9 & 14.2 & 2.3 & 4.8 & $<0.2$ & 5.5 & 1.7 & 9.5 & 74.2 & $<1.0$ & $<2.9$ & 0.39 & 0.17 \\
\hline TBPA & 15 & OSU & DUP & 0.09 & 0.91 & 0.08 & 0.07 & 0.53 & 206.1 & 88.4 & 11.5 & 1.4 & 2.1 & $<0.2$ & 3.1 & 5.3 & 8.4 & 69.7 & $<1.0$ & $<2.0$ & 0.63 & 0.05 \\
\hline TBPR & 16 & OSU & & 0.12 & 1.19 & 0.06 & 0.17 & 0.74 & 363.2 & 51.4 & 21.6 & 3.9 & 9.2 & $<0.2$ & 6.5 & 3.6 & 10.6 & 59.9 & $<1.0$ & $<2.0$ & 1.00 & 0.07 \\
\hline TBPR & 16 & WIS & & & & & & & & & & & & & & & & & & & & \\
\hline TBPA & 17 & OSU & & 0.08 & 0.68 & 0.03 & 0.16 & 0.51 & 224.2 & 32.5 & 12.7 & 3.7 & 6.5 & 50,2 & 5.1 & 2.7 & 10.1 & 31.5 & $\leq 1,0$ & $\leq 2.0$ & 0.54 & 0.13 \\
\hline TBPA & 18 & OSU & & 0.07 & 0.50 & 0.10 & 0.07 & 0.70 & 1797.3 & 26.9 & 22.8 & 2.2 & 6.6 & $<0.2$ & 3.3 & 1.6 & 11.1 & 84.5 & $<1.9$ & $<2.0$ & 0.23 & 0.13 \\
\hline TBPA & 18 & OSU & DUP & & & & & & & & & & & & & & & & & & & \\
\hline TBPA & 19 & OSU & & 0.10 & 1.06 & 0.10 & 0.03 & 0.65 & 107.6 & 27.2 & 8.1 & 2.9 & 3.9 & $<0.2$ & 4.0 & 1.9 & 10.3 & 127.8 & $<1.0$ & $<2.0$ & 0.65 & 0.12 \\
\hline TBPA & 19 & $\bar{W} \mid S$ & & & & & & & & & & & & & & & & & & & & \\
\hline TBPA & 20 & OSU & & 0.10 & 1.07 & 0.11 & 0.03 & 0.67 & 192.4 & 37.4 & 15.4 & 2.7 & 5.2 & $<0.2$ & 5.2 & 2.0 & 15.8 & 147.4 & $<1.0$ & $\leq 2.0$ & $<0.2$ & 0.07 \\
\hline TBPA & 21 & OSU & & 0.09 & 1.02 & 0.06 & 0.08 & 0.63 & 1641.0 & 48.8 & 58.6 & 3.0 & 3.9 & $<0.2$ & 4.6 & 2.3 & 13.4 & 89.0 & $<1.0$ & $<2.0$ & 0.39 & 0.04 \\
\hline TBPA & 22 & OSU & & 0.15 & 1.53 & 0.07 & 0.08 & 0.81 & 2184.4 & 77.4 & 51.7 & 4.8 & 7.8 & 50.2 & 7.1 & 3.6 & 18.2 & 93.0 & $<1,0$ & $<2.0$ & 0.53 & $<0.02$ \\
\hline TBPA & 23 & OSU & & 0.12 & 1.29 & 0.05 & 0.04 & 0.54 & 1635.1 & 34.0 & 32.5 & 3.7 & 3.1 & $<0.2$ & 4.6 & 3.2 & 8.9 & 82.7 & $<1.0$ & $<2.0$ & 0.72 & 0.02 \\
\hline TBPR & 23 & WIS & & & & & & & & & & & & & & & & & & & & \\
\hline TBPA & 24 & OSU & & 0.13 & 1.13 & 0.08 & 0.07 & 0.64 & 1246.8 & 46.8 & 55.1 & 3.4 & 2.8 & $<0.2$ & 4.5 & 2.8 & 14.0 & 103.8 & $<1.0$ & $<2.9$ & 0.66 & 0.03 \\
\hline TBPR & 24 & WIS & & & & & & & & & & & & & & & & & & & & \\
\hline TBPR & 25 & OSU & & 0.11 & 0.85 & 0.08 & 0.09 & 0.84 & 1456.7 & 44.5 & 66.7 & 3.0 & 5.1 & $<0.2$ & 5.5 & 2.2 & 11.1 & 139.5 & $<1.0$ & $<2.0$ & 0.43 & 0.07 \\
\hline TBPA & 26 & OSU & & 0.20 & 1.28 & 0.07 & 0.07 & 1.00 & 16546 & 43.7 & 74.9 & 4.7 & 6.9 & 0.2 & 5.8 & 2.7 & 12.5 & 102.4 & $<10$ & $<2.0$ & 0.66 & 0.04 \\
\hline TBPR & 26 & osu & DUP & & & & & & & & & & & & & & & & & & & \\
\hline TBPB & 27 & OSU & & 0.17 & 1.44 & 0.09 & 0.08 & 0.86 & 2309.5 & 44.6 & 84.1 & 4.7 & 4.5 & $\leq 0.2$ & 5.7 & 3.1 & 10.3 & 96.1 & $<1.0$ & $<2,0$ & 0.49 & 0,03 \\
\hline TBPA & 28 & OSU & & 0.11 & 0.84 & 0.04 & 0.05 & 0.51 & 3413.9 & 136.1 & 37.4 & 3.3 & 5.5 & $<0.2$ & 3.9 & 2.4 & 11.3 & 76.0 & $<1.0$ & $<2.0$ & 0.32 & 0.04 \\
\hline TBPA & 29 & OSU & & 0.09 & 0.88 & 0.10 & 0.10 & 0.91 & 2479.0 & 34.8 & 87.9 & 4.3 & 3.8 & $<0.2$ & 3.4 & 1.6 & 11.0 & 157.6 & $<1.9$ & $<2.0$ & 0.31 & 0.02 \\
\hline TBPR & 29 & osu & DUP & & & & & & & & & & & & & & & & & & & \\
\hline TBPA & 29 & WIS & & 0.08 & 0.84 & 0.09 & 0.09 & 0.69 & 2605.0 & 25.9 & 81.4 & 4.4 & 4.7 & $\leq 0.2$ & 2.2 & 0.9 & 10.3 & 133.9 & & $\leq 2.0$ & & \\
\hline TBPR & 30 & OSU & & 0.11 & 1.02 & 0.06 & 0.08 & 0.77 & 1656.9 & 28.3 & 63.6 & 3.0 & 2.9 & $<0.2$ & 3.3 & 1.7 & 12.0 & 101.4 & $\leq 1.0$ & $<2.0$ & 0.42 & 50.02 \\
\hline
\end{tabular}


Table A,2 cont

\begin{tabular}{|c|c|c|c|c|c|c|c|c|c|c|c|c|c|c|c|c|c|c|c|c|c|c|}
\hline & & & & LEAF & LEAF & LEAF & LEAF & LEAF & LEAF & LEAF & LEAF & LEAF & LEAF & LEAF & LEAF & LEAF & LEAF & LEAF & LEAF & LEAF & LEAF & LEAF \\
\hline SITE/ & & & & $P$ & $k$ & $\mathrm{Ca}$ & $\mathrm{Mg}$ & $\mathrm{N}$ & $\mathrm{Na}$ & $\mathrm{Fe}$ & $\mathrm{Mn}$ & B & $\mathrm{Pb}$ & $\mathrm{Cd}$ & $\mathrm{Ni}$ & $\mathrm{Cr}$ & $\mathrm{Cu}$ & $\mathrm{Zn}$ & $\mathrm{Hg}$ & As & Mo & \\
\hline STUDY & ID & $\angle A B$ & TYYPE & $(\%)$ & $(\%)$ & $(\%)$ & $(\%)$ & $(\%)$ & $(u g / g)$ & $(\mathrm{ug} / \mathrm{g})$ & $(\mathrm{ug} / \mathrm{g})$ & $(u g / g)$ & $(u g / g)$ & $(u g / g)$ & $(u g / g)$ & $(u g / g)$ & $(u g / g)$ & $(u g / g)$ & $(\mathrm{ug} / \mathrm{g})$ & $(u g / g)$ & $(u g / g)$ & $(u g / g)$ \\
\hline TBPR & 1 & OSU & & 0.17 & 1.42 & 0.23 & 0.14 & 1.75 & 110.1 & 50.0 & 41.7 & 13.2 & 5.6 & $<0.2$ & 5.1 & 3.0 & 6.7 & 101.1 & $<1.0$ & $<2.0$ & 1.00 & \\
\hline TBPA & 1 & Wis & & 0.14 & 1.64 & 0.19 & 0.14 & 1.56 & 118.0 & 44.1 & 34.1 & 9.6 & 4.4 & $<0.2$ & 1.8 & 0.6 & 5.6 & 60.0 & & $<2.0$ & & \\
\hline TBPR & 2 & OSU & & 0.12 & 1.46 & 0.22 & 0.27 & 1.80 & 37.0 & 70.9 & 11.0 & 13.5 & 8.8 & $<0.2$ & 4.5 & 3.6 & 6.9 & 57.1 & $<1.0$ & $<2.0$ & 1.60 & 0.06 \\
\hline TBPR & 3 & OSU & & 0.13 & 1.27 & 0.27 & 0.26 & 2.63 & 222.9 & 113.9 & 15.2 & 13.2 & 25.0 & $<0.2$ & 4.6 & 3.5 & 8.6 & $\begin{array}{l}338.4 \\
\end{array}$ & $<1.9$ & $<2.0$ & 1.30 & 0.08 \\
\hline TBPR & 3 & OSU & DUP & 0.12 & 1.28 & 0.21 & 0.24 & 2.53 & 226 & 72.4 & 11.8 & 9.2 & 21.7 & $<0.2$ & 2.7 & 1.8 & 7 & 278.9 & $<1.0$ & $<2.0$ & 0.91 & 0.02 \\
\hline TBPR & 3 & WIS & & & & & & & & & & & & & & & & & & & & \\
\hline TBPA & 4 & OSU & & 0.17 & 1.32 & 0.16 & 0.16 & 2.32 & 89.2 & 63.4 & 13.7 & 10.2 & 16.1 & $<0.2$ & 3.9 & 3.3 & 6.9 & 71.6 & $<1.9$ & $<2.0$ & 0.63 & 0.06 \\
\hline TBPR & 4 & OSU & DUP & & & & & & & & & & & & & & & & & & & \\
\hline TBPR & 4 & WIS & & 0.14 & 1.64 & 0.15 & 0.17 & 2.18 & 121.0 & 62.6 & 12.0 & 6.0 & 16.2 & $<0.2$ & 1.7 & 0.7 & 5.7 & 60.9 & & $\leq 2.0$ & & \\
\hline TBPR & 4 & WIS & DUP & & & & & & & 62.6 & 12.0 & & 16.1 & $<0.2$ & 1.7 & 0.7 & 5.7 & 60.7 & & $<2.0$ & & \\
\hline TBPA & 5 & OSU & & 0.11 & 1.13 & 0.39 & 0.22 & 2.08 & 43.5 & 63.9 & 9.4 & 9.0 & 18.7 & $<0.2$ & 3.7 & 28 & 7.9 & 187.4 & $<1.0$ & $<2.0$ & 1.20 & 0.07 \\
\hline TBPR & 6 & $\overline{\text { OSU }}$ & & 0.16 & 1.30 & 0.32 & 0.34 & 2.18 & 119.0 & 76.3 & 22.9 & 14.2 & 13.3 & $<0.2$ & 5.2 & 5.0 & 9.1 & 59.7 & $<1.0$ & $<2.0$ & 1.00 & 0.24 \\
\hline TBPA & 6 & osu & DUP & 0.15 & 1.38 & 0.26 & 0.32 & 2.37 & 119.4 & 60.6 & 19.1 & 11.5 & 11.0 & $<0.2$ & 3.0 & 2.0 & 7.8 & 50.0 & $<1.0$ & $<2.0$ & 0.78 & 0.10 \\
\hline IBPA & 7 & OSU & & 0.19 & 1.40 & 0.20 & 0.17 & 1.74 & 55.2 & 70.5 & 27.8 & 13.9 & 9.3 & $<0.2$ & 6.6 & 5.2 & 7.0 & 37.5 & $<1.0$ & $<2,0$ & 1.20 & 0.20 \\
\hline TBPA & 8 & OSU & & 0.16 & 1.14 & 0.30 & 0.51 & 2.22 & 83.7 & 68.6 & 29.6 & 15.7 & 72 & $<0.2$ & 5.7 & 4.0 & 10.6 & 51.5 & $<1.0$ & $<2.9$ & 4.80 & 0.31 \\
\hline TBPA & 9 & $\overline{\mathrm{OSU}}$ & & 0.16 & 1.25 & 0.91 & 0.19 & 2.42 & 72.6 & 93.9 & 139.2 & 10.7 & 9.6 & $<0.2$ & 6.6 & 4.5 & 11.9 & 108.8 & $<1.9$ & $<2.0$ & 0.63 & 0.22 \\
\hline TBPR & 9 & OSU & DUP & & & & & & & & & & & & & & & & & & & \\
\hline TBPA & 9 & WIS & & & & & & & & & & & & & & & & & & & & \\
\hline TBPA & 9 & WIS & DUP & & & & & & & & & & & & & & & & & & & \\
\hline TBPA & 10 & OSU & & 0.18 & 1.52 & 0.53 & 0.20 & 1.83 & 47.0 & 85.3 & 47.7 & 11.2 & 10.3 & $<0.2$ & 7.5 & 5.5 & 10.9 & 47.0 & $<1.0$ & $<2.0$ & 1.60 & 0.17 \\
\hline TBPR & 10 & OSU & DUP & & & & & & & & & & & & & & & & & & & \\
\hline TBPR & 11 & OSU & & 0.15 & 1.10 & 1.06 & 0.09 & 1.86 & 52.1 & 60.4 & 75.7 & 8.0 & 11.0 & $<0.2$ & 5.2 & 3.0 & 10.0 & 39.3 & $<1.0$ & $<2.0$ & 0.62 & 0.08 \\
\hline TBPA & 11 & OSU & DUP & & & & & & & & & & & & & & & & & & & \\
\hline TBPA & 12 & 0 Ut & & 0.13 & 1.08 & 0.23 & 0.38 & 1.82 & 85.3 & 73.1 & 16.5 & 10.0 & 6.5 & $<0.2$ & 4.7 & 4.1 & 6.7 & 24.3 & $<1.0$ & $<2.0$ & 0.92 & 0.32 \\
\hline TBPA & 73 & OSU & & 0.15 & 1.23 & 0.23 & 0.37 & 1.42 & 624 & 76.6 & 29.3 & 11.4 & 6.0 & $<0.2$ & 7.2 & 5,1 & 7.2 & 28.3 & $<1.0$ & $<2.0$ & 2.40 & 0.40 \\
\hline TBPA & 14 & OSU & & 0.15 & 1.46 & 0.20 & 0.31 & 1.62 & 85.8 & 86.2 & 21.8 & 8.3 & 3.9 & $<0.2$ & 5.6 & 4.7 & 6.9 & 30.3 & $<1.9$ & $<2.0$ & 1.20 & 0.08 \\
\hline TBPP & 14 & WIS & & & & & & & & & & & & & & & & & & & & \\
\hline TBPR & 15 & OSU & & 0.19 & 1.15 & 0.43 & 0.22 & 1.81 & 47.6 & 58.7 & 30.5 & 9.2 & 3.5 & $<0.2$ & 6.8 & 3.9 & 8.9 & 30.9 & $<1.0$ & $<2.0$ & 1.40 & 0.64 \\
\hline TBPA & 15 & OSU & DUP & & & & & & & & & & & & & & & & & & & \\
\hline TBPR & 16 & OSU & & 0.16 & 1.36 & 0.32 & 0.41 & 2.11 & 49.5 & 76.6 & 36.4 & 15.5 & 3.0 & $<0.2$ & 5.4 & 4.9 & 7.8 & 37.1 & $<1.0$ & $<2.0$ & 2.60 & 0.43 \\
\hline TBPA & 16 & WIS & & 0.15 & 1.71 & 0.28 & 0.44 & 2.02 & 71.0 & 64.8 & 34.2 & 9.7 & 2.5 & $<0.2$ & 2.6 & 1.4 & 6.6 & 21.5 & & $<2.0$ & & \\
\hline TBPA & 17 & OSU & & 0.13 & 0.90 & 0.18 & 0.56 & 1.73 & 53.6 & 53.0 & 36.0 & 9.3 & $\leq 2.0$ & $\leq 0.2$ & 3.6 & 3.2 & 6.4 & 28.3 & $<1,0$ & $<2.0$ & 1.20 & 0.29 \\
\hline TBPA & 18 & OSU & & 0.17 & 1.34 & 0.42 & 0.18 & 2.02 & 565.2 & 65.0 & 51.4 & 13.0 & 3.2 & $<0.2$ & 5.2 & 3.4 & 8.5 & 32.2 & $<1.0$ & $<2.0$ & 0.89 & \\
\hline TBPP & 18 & osu & DUP & 0.16 & 1.46 & 0.41 & 0.19 & 1.96 & 631.7 & 59.4 & 49.3 & 11.4 & 2.7 & $<0.2$ & 3.6 & 1.9 & 7.4 & 22.6 & $<1.0$ & $<2.0$ & 0.64 & 0.13 \\
\hline TBPR & 19 & OSU & & 0.16 & 1.32 & 0.43 & 0.10 & 2.09 & 48.6 & 65.3 & 17.4 & 8.4 & 5.2 & $<0.2$ & 7.3 & 4.2 & 9.5 & 48.1 & $<1.0$ & $<2.0$ & 1.50 & 0.19 \\
\hline TBPA & 19 & WIS & & & & & & & & & & & & & & & & & & & & \\
\hline TBPR & 20 & OSU & & 0.14 & 1.42 & 0.59 & 0.12 & 1.52 & 52.2 & 71.3 & 24.4 & 6.6 & 4.8 & $<0,2$ & 7.2 & 4.7 & 7.6 & 87.7 & $\leq 1.0$ & $\leq 2.0$ & 0.60 & 0.17 \\
\hline TBPR & 21 & osu & & 0.20 & 1.29 & 0.33 & 0.22 & 2.75 & 199.6 & 99.4 & 149.7 & 11.0 & 3.2 & $<0.2$ & 8.1 & 3.6 & 10.9 & 27.8 & $<1.0$ & $<2.0$ & 1.40 & 0.07 \\
\hline TBPR & 22 & OSU & & 0.23 & 1.34 & 0.30 & 0.21 & 2.96 & 194.4 & 108.5 & 108.7 & 14.7 & 3.0 & 0.2 & 5.8 & 4.3 & 13.3 & 30.7 & $<1.0$ & $<2.0$ & 1.50 & 0.08 \\
\hline & 231 & OSU & & 0.23 & 1.36 & 0.37 & 0.17 & 2.48 & 224.1 & 123.5 & 125.9 & 11.4 & 4.5 & $<0.2$ & 6.2 & 3.2 & 8.9 & 46.5 & $<1.0$ & $<2.0$ & 1.10 & 0.09 \\
\hline TBPR & 23 & WIS & & & & & & & & & & & & & & & & & & & & \\
\hline TBPR & 24 & OSU & & 0.22 & 1.34 & 0.31 & 0.19 & 2.73 & 96.2 & 96.9 & 97.0 & 12.2 & 4.2 & 0.3 & 4.4 & 3.7 & 9.9 & 49.0 & $<1.0$ & $<2.0$ & 1.50 & 0.06 \\
\hline TBPR & 24 & WIS & & 0.19 & 1.79 & 0.26 & 0.20 & 2.45 & 142.0 & 75.5 & 87.7 & 6.8 & 1.4 & $<0.2$ & 2.3 & 0.6 & 77.5 & 18.8 & & $\leq 2.0$ & & \\
\hline TBPR & 25 & OSU & & 0.18 & 1.26 & 0.40 & 0.17 & 2.70 & 79.6 & 157.1 & 161.4 & 14.3 & 2.9 & $<0.2$ & 4.6 & 3.7 & 9.3 & 29.4 & $<1.0$ & $<2.0$ & 1.80 & 0.11 \\
\hline TBPA & 26 & OSU & & 0.27 & 1.60 & 0.27 & 0.16 & 2.97 & 163.9 & 134.4 & 142.2 & 11.8 & 4.7 & $<0.2$ & 5.4 & 3.6 & 13.5 & 32.1 & $<1.0$ & $<2.0$ & 1.40 & 0.08 \\
\hline TBPR & 26 & OSU & DUP & 0.25 & 1.65 & 0.25 & 0.17 & 2.92 & 174.9 & & & 9.6 & 3.9 & & 4.4 & 1.9 & 12.1 & 28.1 & $\leq 1.0$ & $<2.0$ & 1.00 & 0.04 \\
\hline TBPR & 27 & OSU & & 0.21 & 1.46 & 0.37 & 0.18 & 2.81 & 460.3 & 140.5 & 144.8 & 15.0 & 3.9 & $<0.2$ & 4.4 & 2.7 & 10.5 & 28.1 & $<1.0$ & $<2.0$ & 1.00 & 0.10 \\
\hline TBPA & 28 & OSU & & 0.24 & 1.53 & 0.30 & 0.21 & 2.57 & 577.8 & 123.5 & 118.3 & 15.6 & 8.4 & $<0.2$ & 14.4 & 3.1 & 12.7 & 26.0 & $<1.0$ & $<2.0$ & 1.20 & 0.14 \\
\hline TBPR & 29 & OSU & & 0.17 & 1.16 & 0.63 & 0.29 & 2.89 & 389.4 & 127.7 & 216.3 & 21.7 & 8.4 & $<0.2$ & 3.1 & 2.8 & 9.2 & 41.3 & $\leq 1.9$ & $<2.9$ & 0.64 & 0.07 \\
\hline TBPR & 29 & OSU & DUP & & & & & & & & & & & & & & & & & & & \\
\hline TBPA & 29 & $\overline{W I S}$ & & & & & & & & & & & & & & & & & & & & \\
\hline TRP & 30 & OSU & & 0.18 & 126 & 0.43 & 0.21 & 2.29 & 291.1 & 68.2 & 165.4 & 14.4 & 7.2 & $<0.2$ & 4.7 & 2.9 & 8.7 & 23.5 & $<1.0$ & $<2.0$ & 1.10 & 0.08 \\
\hline
\end{tabular}


Table A.2 cout

\begin{tabular}{|c|c|c|c|c|c|c|c|c|c|c|c|c|c|c|c|c|c|c|c|c|c|c|}
\hline & & & & RHIZ & RHIZ & RHIZ & RHIZ & RHIZ & RHIZ & RHIZ & RHIZ & RHIZ & RHIZ & RHIZ & RHIZ & RHIZ & $\mathrm{RHIZ}$ & RHIZ & RHIZ & RHIZ & RHIZ & RHIZ \\
\hline SITE/ & & & & $P$ & $\mathrm{~K}$ & $\mathrm{Ca}$ & $\mathrm{Mg}$ & $\mathrm{N}$ & $\mathrm{Na}$ & $\mathrm{Fe}$ & $\mathrm{Mn}$ & B & $\mathrm{Pb}$ & $\mathrm{Cd}$ & $\mathrm{Ni}$ & $\mathrm{Cr}$ & $\mathrm{Cu}$ & $\mathrm{Zn}$ & $\mathrm{Hg}$ & As & Mo & $\mathrm{Se}$ \\
\hline STUDY & ID & $\angle A B$ & TYPE & (\%) & $(\%)$ & $(\%)$ & $\langle \%\rangle$ & (\%) & $(u g / g)$ & $(u g / g)$ & $(u g / g)$ & $(u g / g)$ & $(u g / g)$ & $(u g / g)$ & $\langle(\mathrm{ug} / \mathrm{g}\rangle$ & $(u g / g)$ & (ug/g) & $(\mathrm{ug} / \mathrm{g})$ & $(u g / g)$ & $(u g / g)$ & $(u g / g)$ & $(u g / g)$ \\
\hline TBPR & 1 & OSU & & 0.04 & 0.75 & 0.04 & 0.08 & 0.34 & 982.1 & 306.9 & 39.6 & 4.8 & 102.0 & $<0.2$ & 8.3 & 8.0 & 20.4 & 218.7 & $<1.0$ & $<2.0$ & 1.80 & $<0.02$ \\
\hline TBPA & 1 & WIS & & 0.04 & 0.80 & 0.04 & 0.08 & 0.22 & 972.0 & 311.1 & 57.4 & 1.8 & 95.6 & 0.4 & 8.1 & 7.1 & 23.0 & 289.9 & & $<2.0$ & & \\
\hline TBPR & 2 & OSU & & 0.03 & 0.79 & 0.05 & 0.07 & 0.67 & 779.5 & 1001.1 & 13.6 & 5.0 & 434.2 & 0.7 & 6.2 & 8.9 & 102.9 & 576.6 & $<1.0$ & $\leq 2.0$ & 1.10 & $<0.02$ \\
\hline TBPR & 3 & OSU & & 0.05 & 0.88 & 0.05 & 0.09 & 0.62 & 1139.2 & 303.0 & 19.4 & 4.5 & 458.9 & 0.7 & 6.7 & 3.9 & 47.4 & 697.8 & $<1.0$ & $<2.0$ & 0.84 & 0.02 \\
\hline TBPR & 3 & OSU & DUP & & & & & & & & & & & & & & & & & & & \\
\hline TBPR & 3 & WIS & & & & & & & & & & & & & & & & & & & & \\
\hline TBPR & 4] & OSU & & 0.04 & 0.40 & 0.03 & 0.07 & 0.76 & 898.7 & 1136.3 & 19.3 & 3.7 & 726.4 & $<0.2$ & 4.6 & 6.2 & 53.8 & 375.3 & $<1.0$ & $<2.0$ & 0.44 & 0.03 \\
\hline TBPR & 4 & osu & DUP & 0.02 & 0.40 & 0.03 & 0.07 & 0.73 & 879.4 & 260.7 & 17.8 & 3.3 & 875.7 & $<0.2$ & 4.7 & 4.9 & 55.3 & 384.6 & $<1.0$ & $<2.0$ & $<0.2$ & 0.04 \\
\hline TBPR & 4 & WIS & & & & & & & & & & & & & & & & & & & & \\
\hline TBPR & 4 & WIS & DUP & & & & & & & & & & & & & & & & & & & \\
\hline TBPA & 5 & OSU & & 0.03 & 0.75 & 0.05 & 0.07 & 0.59 & 805.4 & 200.5 & 8.9 & 2.8 & 286.3 & 0.5 & 8.6 & 5.8 & 54.5 & 462.2 & $<1.0$ & $<2.0$ & 0.91 & 0.04 \\
\hline $\mathrm{BBPA}$ & 6 & OSU & & 0.05 & 0.69 & 0.06 & 0.15 & 0.46 & 973.7 & 649.8 & 23.2 & 5.4 & 196.2 & 0.5 & 6.6 & 19.8 & 143.8 & 270.2 & $\leq 1.9$ & $<2.0$ & 0.93 & 0.12 \\
\hline TBPA & 6 & OSU & DUP & & & & & & & & & & & & & & & & & & & \\
\hline TBPA & 7 & OSU & & 0.05 & 0.87 & 0.05 & 0.11 & 0.41 & 1453.7 & 365.9 & 28.6 & 4.2 & 145.9 & $<0.2$ & 10.0 & 6.7 & 50.5 & 336.2 & $<1.0$ & $<2.0$ & 0.96 & 0.07 \\
\hline TBPA & 8 & oSU & & 0.06 & 0.92 & 0.06 & 0.20 & 0.34 & 1956.1 & 399.4 & 26.0 & 6.7 & 51.7 & 0.5 & 7.3 & 12.6 & 49.8 & 92.2 & $<1.0$ & $<2.0$ & 1.60 & 0.03 \\
\hline TBPA & 9 & OSU & & 0.05 & 1.04 & 0.09 & 0.11 & 0.34 & 1012.2 & 213.6 & 58.7 & 4.8 & 32.0 & 1.3 & 11.4 & 9.6 & 41.6 & 152.0 & $<1.0$ & $<2.0$ & 0.60 & $<0.02$ \\
\hline TBPR & 9 & OSU & DUP & 0.05 & 0.95 & 0.08 & 0.10 & 0.40 & 830.1 & 130.1 & 48.0 & 4.6 & 36.2 & 1.1 & 12.1 & 9.2 & 39.4 & 142.7 & $<1.0$ & $<2.0$ & 0.54 & $\leq 0.02$ \\
\hline TBPR & 9 & WIS & & & & & & & & & & & & & & & & & & & & \\
\hline TBPA & 9 & & DUP & & & & & & & & & & & & & & & & & & & \\
\hline TBPR & 10 & OSU & & 0.05 & 0.79 & 0.07 & 0.12 & 0.42 & 1172.3 & 768.3 & 47.8 & 4.4 & 93.0 & 0.9 & 6.5 & 5.4 & 34.4 & 205.4 & $\leq 1.0$ & 3.7 & 0.86 & 0.03 \\
\hline TBPR & 10 & osu & DUP & & & & & & & & & & & & & & & & & & & \\
\hline TBPR & 11 & OSU & & 0.04 & 0.45 & 0.31 & 0.14 & 0.44 & 897.6 & 483.3 & 28.9 & 5.1 & 69.7 & 3.8 & 5.2 & 8.0 & 31.7 & 226.7 & $<1.0$ & $<2.0$ & 0.54 & $0.0^{\prime}$ \\
\hline TBPR & 11 & OSU & DUP & & & & & & & & & & & & & & & & & & & \\
\hline TBPR & 12 & OSU & & 0.06 & 0.61 & 0.04 & 0.17 & 0.32 & 502.2 & 329.5 & 94 & 4.6 & 9.3 & 0.6 & 3.6 & 7.7 & 26.4 & 100.9 & $\leq 1.0$ & $\leq 2,0$ & 1.00 & 0.03 \\
\hline TBPR & 13 & OSU & & 0.11 & 0.93 & 0.04 & 0.19 & 0.29 & 261.2 & 267.7 & 10.0 & 5.3 & 5.8 & 0.5 & 4.7 & 5.5 & 22.0 & 90.2 & $<1.0$ & $<2.0$ & 1.20 & $<0.02$ \\
\hline TBPA & 14 & OSU & & 0.04 & 0.67 & 0.06 & 0.16 & 0.59 & 1152.1 & 512.6 & 14.4 & 4.4 & 15.9 & 0.2 & 3.9 & 7.9 & 41.1 & 76.2 & $<1.0$ & $<2.0$ & 0.79 & $<0.02$ \\
\hline TBPR & 14 & WIS & & 0.04 & 0.68 & 0.06 & 0.16 & 0.52 & 1096.0 & 765.6 & 15.6 & 2.5 & 25.6 & 0.4 & 3.0 & 5.9 & 45.7 & 73.1 & & $<2.0$ & & \\
\hline TBPR & 15 & OSU & & 0.05 & 0.45 & 0.04 & 0.09 & 0.27 & 439.7 & 222.7 & 20.7 & 2.9 & 18.5 & $<0.2$ & 10.0 & 4.9 & 11.7 & 78.6 & $\leq 1.9$ & $<2.0$ & 0.84 & 0.03 \\
\hline TBPR & 15 & osu & DUP & 0.05 & 0.42 & 0.04 & 0.09 & 0,23 & 420.4 & 290.4 & 20.7 & 2.5 & 20.4 & $\leq 0.2$ & 10.7 & 5.1 & & & & & 0.87 & 0. \\
\hline TBPR & 16 & OSU & & 0.09 & 0.82 & 0.05 & 0.19 & 0.37 & 2963.0 & 313.9 & 19.8 & 3.0 & 21.8 & 0.4 & 6.3 & 9.7 & 32.7 & 53.8 & $<1.0$ & $<2.0$ & 1.40 & $<0.02$ \\
\hline TBPR & 16 & WIS & & & & & & & & & & & & & & & & & & & & \\
\hline $3 P R$ & 17 & OSU & & 0.11 & 1.14 & 0.03 & 0.12 & 0.31 & 884.1 & 304.5 & 15.6 & 4.8 & 11.3 & $\leq 0.2$ & 4.9 & 6.3 & 14.2 & 49.6 & $<1.0$ & $\leq 2.0$ & 1.00 & $\leq 0.02$ \\
\hline $\mathrm{PB}$ & 18 & osu & & 0.07 & 0.56 & 0.05 & 0.10 & 0.41 & 2306.5 & 808.0 & 27.7 & 2.6 & 18.8 & 0.9 & 7.6 & 6.1 & 15.8 & 65.2 & $<1.9$ & $<2.9$ & 0.67 & 0.02 \\
\hline PPR & 18 & osu & DUP & & & & & & & & & & & & & & & & & & & \\
\hline TBPR & 19 & OSU & & 0.06 & 0.63 & 0.05 & 0.07 & & 358.6 & 387.3 & 17.1 & 3.6 & 139 & 0.4 & 4.7 & 12.1 & & 1250 & $<1.0$ & $<2.0$ & 0.90 & 0.02 \\
\hline TBPA & 19 & WIS & & 0.06 & & 0.05 & 0.07 & & 363.0 & 284 & & 5.0 & & 0.5 & 2.7 & 9.0 & 46.9 & 122. & & $\leq 2.0$ & & \\
\hline TBPR & 201 & OSU & & 0.03 & 0.36 & 0.05 & & & 438.8 & 293.3 & 14.9 & 1.9 & & & & 6.6 & 20.9 & & $<1.0$ & $<2.0$ & 0.55 & $<0.02$ \\
\hline TBPA & 21 & OSU & & 0.04 & 0.40 & 0.03 & 0.05 & 30 & 2512.4 & 140.0 & 89.1 & 5.0 & 5.4 & 1.6 & 3.1 & 2.6 & 9.1 & 62.9 & $<1.0$ & $\leq 2.0$ & 0.31 & $\leq 0.02$ \\
\hline $\mathrm{PR}$ & 22 & OSU & & 0.03 & 0.36 & 0.04 & & & 3118.8 & .63 .2 & 107.2 & 4.3 & 21.9 & 1.8 & 3.1 & 1.6 & 12.1 & & $\leq 1.0$ & $<2.0$ & 0.59 & $<0.0$ \\
\hline TBPR & $23 \mathrm{C}$ & OSU & & & & & & & & & & & & & & & & & $<1$. & $<2.0$ & 0.27 & $<0.0$ \\
\hline TBPR & 23 & WIS & & 0.03 & 0.47 & 0.03 & 0.05 & 0.02 & 2506.0 & & 155.2 & 1.4 & 12.8 & 2.0 & 4.1 & 1.1 & 12.9 & 144.0 & & $<2.0$ & & \\
\hline $8 \pi$ & 24 & OSU & & 0.05 & 0.71 & 0.03 & 0.05 & 0.31 & 2962.1 & 316.4 & 64.6 & 4.1 & 7.4 & 0.7 & 3.6 & 2.1 & 10.4 & 47.7 & $<1.0$ & $<2.0$ & 0.41 & $\leq 0.02$ \\
\hline$P R$ & $24[1$ & WIS & & & & & & & & & & & & & & & & & & & & \\
\hline TBPA & 251 & OSU & & 0.05 & 0.54 & 0.03 & 0.07 & 0.31 & 3530.5 & 1009.2 & 177.7 & 5.4 & 23.8 & 2.0 & 8.1 & 10.9 & 15.2 & 88.4 & & $\leq 2.0$ & 0.68 & $\leq 0.02$ \\
\hline & $\frac{50}{26}$ & OSU & & 0.07 & 0.45 & 0.04 & 0.06 & 0.40 & 2773.7 & 204.6 & 98.2 & 4.3 & 232.2 & 1.8 & 14.9 & 2.7 & 13.7 & 94.7 & $\leq 1.0$ & $<2.0$ & 0.48 & $\leq 0.02$ \\
\hline $\mathrm{PA}$ & 26.0 & osu & DUP & & & & & & & & & & & & & & & & & & & \\
\hline PR & 2710 & $\mathrm{OSU}$ & & 0.04 & 0.39 & 0.06 & 0.10 & 0. & & & 118.3 & 5.6 & & & & & 14.5 & & & $\leq 2.0$ & 0.59 & $\leq 0.02$ \\
\hline & 2810 & osu & & 0.03 & 0.30 & 0.06 & 0.12 & 0.31 & 4747.9 & 1941.2 & 62.4 & 5.9 & 28.0 & 0.8 & 4.9 & 5.8 & 17.4 & 104. & $<1.0$ & 5.1 & 0.47 & $<0.02$ \\
\hline & $29 / 0$ & OSU & & 0.04 & 0.64 & 0.08 & 0.14 & 0.42 & 5016.9 & 1053.9 & 91.4 & 9.0 & 30.6 & 2.7 & 5.3 & 4.0 & & 168.5 & & 2.4 & 0.42 & $<0.02$ \\
\hline & 29 & osu & DUP & 0.04 & 0.67 & 0.08 & 0.13 & 0.45 & 4868.7 & 76.2 & 60.8 & 7.6 & 30.0 & 2.6 & 5.0 & 3.1 & 18.7 & 154.3 & $\leq 1.0$ & 2.3 & 0.3 & 0.04 \\
\hline BPA & $29 \sqrt{1}$ & & & & & & & & & & & & & & & & & & & & & \\
\hline $\mathrm{PH}$ & 3010 & osu & & 0.05 & 0.33 & 0.08 & & & 4304 & 3048 & 121.7 & & 67.3 & 0.6 & 11.0 & 9.2 & 7.81 & 158.0 & & & .58 & 0.02 \\
\hline
\end{tabular}


Table A.Z cont.

\begin{tabular}{|c|c|c|c|c|c|c|c|c|c|c|c|c|c|c|c|c|c|c|c|c|c|c|}
\hline & & & & BOOT & ROOT & ROOT & ROOT & ROOT & ROOT & ROOT & ROOT & ROOT & ROOT & ROOT & ROOT & ROOT & ROOT & ROOT & ROOT & ROOT & ROOT & ROOT \\
\hline SITEI & & & & $\mathrm{P}$ & $\mathrm{K}$ & $\mathrm{Ca}$ & $\overline{M g}$ & $\mathrm{~N}$ & $\mathrm{Na}$ & $\mathrm{Fe}$ & $\mathrm{Mn}$ & $B$ & $\mathrm{~Pb}$ & $\overline{c d}$ & $\mathrm{Ni}$ & $\mathrm{Cr}$ & $\mathrm{Cu}$ & $\mathrm{Zn}$ & $\mathrm{Hg}$ & As & $\mathrm{Mo}$ & $\mathrm{Se}$ \\
\hline STUDY & ID & $\angle A B$ & TYPE & (\%) & $(\%)$ & $(\%)$ & (\%) & (\%) & $(u g / g)$ & $(u g / g)$ & $(\mathrm{ug} / \mathrm{g})$ & $(\mathrm{ug} / \mathrm{g})$ & $(u g / g)$ & $(\mathrm{ug} / \mathrm{g})$ & $(u g / g)$ & $(u g / g)$ & $(u g / g)$ & $(u g / g)$ & $(u g / g)$ & $(u g / g)$ & $(u g / g)$ & $(u g / g)$ \\
\hline TBPR & 1 & OSU & & 0.03 & 0.34 & 0.10 & 0.07 & & 1305.1 & 406.8 & 60.0 & 6.3 & 268.7 & $<0.9$ & 5.3 & 6.3 & 112.9 & 497.8 & $<4.5$ & $<9.1$ & $<0.91$ & \\
\hline IBPR & & WIS & & & & & & & & & & & & & & & & & & & & \\
\hline TBPR & 2 & OSU & & 0.06 & 0.47 & 0.20 & 0.11 & & 1678.3 & 7641.3 & 76.8 & 11.5 & 3400.3 & 3.8 & 48.2 & 90.0 & 782.9 & 2498.1 & $<4.5$ & $<9.1$ & $\leq 0.91$ & \\
\hline TBPR & 3 & Osu & & & & & & 1.72 & & & & & & & & & & & & & & \\
\hline TBPA & 3 & OSU & DUP & & & & & & & & & & & & & & & & & & & \\
\hline TBPR & 3 & WIS & & & & & & & & & & & & & & & & & & & & \\
\hline TBPA & 4 & OSU & & & & & & & & & & & & & & & & & & & & \\
\hline TBPR & 4 & OSU & DUP & & & & & & & & & & & & & & & & & & & \\
\hline TBPR & 4 & WIS & & & & & & & & & & & & & & & & & & & & \\
\hline TBPR & 4 & WIS & DUP & & & & & & & & & & & & & & & & & & & \\
\hline TBPR & 5 & OSU & & & & & & 1.42 & & & & & & & & & & & & & & \\
\hline TBPR & 6 & OSU & & & & & & & & & & & & & & & & & & & & \\
\hline TBPR & 6 & osu & DUP & & & & & & & & & & & & & & & & & & & \\
\hline TBPA & 7 & OSU & & & & & & 1.36 & & & & & & & & & & & & & & \\
\hline TBPA & 8 & OSU & & 0.07 & 0.46 & 0.22 & 0.25 & & 2014.6 & 2719.0 & 184.9 & 14.9 & 365.8 & 2.9 & 7.7 & 62.0 & 432.1 & 260.0 & $\leq 5.0$ & $\leq 10.0$ & $\leq 1.0$ & \\
\hline TBPR & 9 & OSU & & 0.04 & 0.58 & 0.21 & 0.13 & & 1529.3 & 2257.0 & 76.9 & 13.7 & 177.3 & 9.0 & 12.2 & 42.4 & 288.7 & 364.3 & $<3.3$ & $<6.7$ & $<0.67$ & \\
\hline TBPA & 9 & Osu & DUP & & & & & & & & & & & & & & & & & & & \\
\hline TBPA & 9 & WIS & & & & & & & & & & & & & & & & & & & & \\
\hline TBPR & 9 & WIS & DUP & & & & & & & & & & & & & & & & & & & \\
\hline TBPR & 10 & OSU & & 0.04 & 0.57 & 0.22 & 0.15 & & 1480.3 & 3634.0 & 91.6 & 12.0 & 524.2 & 2.2 & 19.8 & 23.7 & 217.9 & 488.1 & $\leq 3.8$ & 10.7 & 0.92 & \\
\hline TBPR & 10 & OSU & DUP & & & & & & & & & & & & & & & & & & & \\
\hline TBPA & 11 & OSU & & 0.07 & 0.24 & 0.63 & 0.17 & & 1327.0 & 4868.0 & 201.5 & 18.6 & 1567.0 & 11.1 & 18.7 & 32.5 & 334.7 & 574.9 & $<5.0$ & 11.2 & $\leq 1.0$ & \\
\hline TBPR & 11 & OSU & DUP & & & & & & & & & & & & & & & & & & & \\
\hline TBPR & 12 & OSU & & 0.05 & 0.43 & 0.15 & 0.26 & & 829.4 & 1809.6 & 36.6 & 12.2 & 71.8 & 5.5 & 9.4 & 20.8 & 147.7 & 267.5 & $\leq 3.6$ & $<7.1$ & $<0.71$ & \\
\hline TBPA & 13 & OSU & & & & & & & & & & & & & & & & & & & & \\
\hline TBPR & 14 & OSU & & 0.07 & 0.45 & 0.21 & 0.29 & & 1991.7 & 3449.5 & 87.5 & 14.3 & 210.6 & 2.5 & 11.9 & 51.6 & 457.1 & 342.8 & $<4.5$ & $<9.1$ & $<0.91$ & \\
\hline TBPR & 14 & WIS & & & & & & & & & & & & & & & & & & & & \\
\hline TBPR & 15 & OSU & & 0.06 & 0.46 & 0.20 & 0.13 & & 1674.4 & 2852.2 & 77.0 & 15.2 & 41.4 & 4.2 & 9.6 & 14.5 & 103.8 & 144.6 & $<3.1$ & $<6.2$ & 0.76 & \\
\hline TBPR & 15 & OSU & DUP & & & & & & & & & & & & & & & & & & & \\
\hline TBPR & 16 & OSU & & 0.07 & 0.54 & 0.14 & 0.23 & & 1743.3 & 3943.2 & 84.9 & 14.3 & 86.9 & 1.8 & 15.6 & 38.5 & 158.9 & 191.3 & $<4.5$ & $<9.1$ & 2.00 & \\
\hline TBPR & 16 & WIS & & & & & & & & & & & & & & & & & & & & \\
\hline TBPR & 17 & OSU & & 0.06 & 0.58 & 0.12 & 0.26 & & 1493.7 & 1355.9 & 48.9 & 11.6 & 87.7 & 1.3 & 12.0 & 21.2 & 127.8 & 144.3 & $\leq 3.1$ & $\leq 6.2$ & $<0.6$ & \\
\hline TBPR & 18 & OSU & & & & & & & 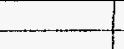 & & & & - & & & & & & & & & \\
\hline$\frac{T B P A}{T B P A}$ & 18 & osu & DUP & 0.05 & $\overline{0.41}$ & 029 & 0.10 & & & & & & & & & & & & & & & \\
\hline$\frac{\text { TBPA }}{\text { TBPR }}$ & $\frac{19}{10}$ & $\frac{\text { OSU }}{\text { WIS }}$ & & & & & & & 1306.6 & 1832.5 & 70.9 & 13.9 & 59.2 & 5.6 & 7.0 & 71.9 & 341.4 & 303.4 & $<3.6$ & $<7.1$ & $<0.71$ & \\
\hline$\frac{T B P A}{T B P A}$ & $\frac{19}{20}$ & WIS & & & & & & 0.92 & & & & & & & & & & & & & & \\
\hline TBPA & 21 & OSU & & 0.04 & 0.11 & 0.19 & 0.12 & 1.07 & 1794.4 & 1024.4 & 53.1 & 21.1 & 21.5 & 11.5 & 4.8 & 4.4 & 79.9 & 227.0 & $<1.0$ & 2.6 & 0.42 & \\
\hline TBPA & 22 & OSU & & 0.04 & 0.19 & 0.18 & 0.15 & 1.06 & 3884.1 & 1459.7 & 40.3 & 17.3 & 32.7 & 154 & 4.3 & 5.5 & 100.1 & 251.3 & $<1.0$ & 3.6 & 0.85 & 0.03 \\
\hline TBPA & 23 & OSU & & 0.05 & 0.18 & 0.16 & 0.15 & 1.13 & 3456.9 & 2154.4 & 42.8 & 16.9 & 26.0 & 7.4 & 5.0 & 5.7 & 93.4 & 410.6 & $<1.9$ & 4.1 & 0.63 & $<0.02$ \\
\hline TBPR & 23 & WIS & & & & & & & & & & & & & & & & & & & & \\
\hline TBPA & 24 & OSU & & 0.07 & 0.38 & 0.18 & 0.19 & 0.99 & 4267.2 & 3411.1 & 67.5 & 20.1 & 39.2 & 10.8 & 7.3 & 5.7 & 62.9 & 328.8 & $\leq 1.9$ & 7.7 & 0.69 & $\leq 0.0$ \\
\hline TBPR & 24 & & & & & & & & & & & & & & & & & & & & & \\
\hline TBPR & 25 & OSU & & 0.07 & 0.16 & 0.18 & 0.20 & 1.09 & 2552.9 & 6809.0 & 55.8 & 12.9 & 94.1 & 29.6 & 7.0 & 12.4 & 142.0 & 412.3 & $\leq 1.0$ & 12.2 & 1.00 & $<0.0$ \\
\hline $\begin{array}{l}\text { TBPR } \\
\text { TBPRR }\end{array}$ & $\begin{array}{l}26 \\
26 \\
\end{array}$ & $\begin{array}{l}\text { OSU } \\
\text { osu }\end{array}$ & DUP & 0.06 & 0.18 & 0.19 & 0.13 & 1.23 & 2010.3 & 23986 & 50.9 & 26.4 & 68.8 & 51.9 & 7.4 & 9.2 & 108.8 & 352.2 & $\leq 1.0$ & 5.9 & 0.98 & $\leq 0.0$ \\
\hline TBPR & 27 & oSu & & 0.05 & 0.28 & 0.17 & 0.14 & 1.09 & 4056.7 & 3999.2 & 43.6 & 24.9 & 129.7 & 2.5 & 10.3 & 14.6 & 133.3 & 208.0 & $<1.0$ & 11.0 & 1.70 & \\
\hline TBPR & 28 & $\mathrm{OSU}$ & & 0.06 & 0.20 & 0.17 & 0.16 & 0.84 & 4577.0 & 9471.6 & 39.1 & 20.5 & 221.1 & 7.0 & 10.5 & 12.5 & 238.9 & 499.2 & $<1.0$ & 34.8 & 1.40 & $\leq 0.0$ \\
\hline TBPR & 29 & osu & & 0.06 & 0.18 & 0.24 & 0.17 & 1.27 & 2309.1 & 6379.0 & 103.7 & 30.3 & 316.0 & 11.7 & 11.2 & 18.1 & 240.3 & 450.8 & $<1.9$ & 17.1 & 0.22 & \\
\hline TBPA & 29 & osu & DUP & & & & & & & & & & & & & & & & & & & \\
\hline TBPA & 29 & WIS & & & & & & & & & & & & & & & & & & & & \\
\hline זBPR & 30 & OSU & & 0.09 & 0.15 & 0.22 & 0.13 & 0.94 & 2638.6 & 9385.2 & 115.4 & 25.4 & 338.8 & 0.2 & 6.2 & 7.9 & 155.7 & 479.1 & $<1.0$ & $\begin{array}{l}33.9 \\
\end{array}$ & 0.20 & \\
\hline
\end{tabular}


Table A.3 Results of plant tissul analyses for Decomber samples

\begin{tabular}{|c|c|c|c|c|c|c|c|c|c|c|c|c|c|c|c|c|c|c|c|c|c|c|}
\hline & & & & STEM & STEM & STEM & STEM & STEM & STEM & STEM & STEM & STEM & STEM & STEM & STEM & STEM & STEM & STEM & STEM & STEM & STEM & STEM \\
\hline SITE/ & & & & $\mathrm{P}$ & $\mathrm{K}$ & $\mathrm{Ca}$ & $\mathrm{Mg}$ & $\mathrm{N}$ & $\mathrm{Na}$ & $\mathrm{Fe}$ & $\overline{M n}$ & $\bar{B}$ & $\mathrm{~Pb}$ & $\mathrm{Cd}$ & $\mathrm{Ni}$ & $\mathrm{Cr}$ & $\mathrm{Cu}$ & $2 n$ & $\mathrm{Hg}$ & As & Mo & $\mathrm{Se}$ \\
\hline STUDY & ID & $\angle A B$ & TYPE & (\%) & $(\%)$ & (\%) & (\%) & $(\%)$ & $(\mathrm{ug} / \mathrm{g})$ & $(u g / g)$ & $(u g / g)$ & $(u g / g)$ & $(\mathrm{ug} / \mathrm{g})$ & $(\mathrm{ug} / \mathrm{g})$ & $(u g / g)$ & $(\mu \mathrm{g} / \mathrm{g})$ & $\langle u g / g)$ & $(u g / g)$ & $(u g / g)$ & $(\mathrm{ug} / \mathrm{g})$ & $(u g / g)$ & $(u g / g)$ \\
\hline TBPA & 1 & OSU & & 0.01 & 0.15 & 0.05 & 0.03 & 0.40 & 726.8 & 51.2 & 18.9 & 1.7 & 12.4 & 0.2 & $\begin{array}{r}4.0 \\
\end{array}$ & $\begin{array}{r}1.2 \\
\end{array}$ & $\quad 3.2$ & $\begin{array}{r}98.9 \\
\end{array}$ & $<1.0$ & $-\leq 2.0$ & & \\
\hline TBPR & 2 & OSU & & 0.01 & 0.16 & 0.03 & 0.04 & 0.24 & 524.1 & 40.8 & 2.1 & 2.9 & 15.1 & 0.2 & 3.6 & 1.5 & 2.4 & 222.9 & $<1.0$ & $<2.0$ & 0.20 & \\
\hline TBPR & 2 & OSU & DUP & 0.01 & 0.16 & 0.03 & $0 . \overline{04}$ & 0.25 & 523.1 & 38.7 & 1.7 & 1.1 & 14.5 & $\leq 0.2$ & 3.8 & 0.9 & 2.2 & 204.6 & $<1.0$ & & $\leq 0.2$ & \\
\hline TBPA & 2 & WIS & & & & & & & & & & & & & & & & & & & & \\
\hline TBPR & 3 & OSU & & 0.01 & 0.13 & 0.04 & 0.03 & 0.39 & 351.6 & 40.3 & 3.7 & 2.3 & 26.9 & 0.2 & 1.8 & 1.2 & 2.9 & 184.3 & $\leq 1.0$ & $\leq 2.9$ & $\frac{0.23}{0.03}$ & $\leq 0.0$ \\
\hline TBPR & $\overline{4}$ & OSU & & 0.01 & 0.07 & 0.05 & 0.05 & 0.37 & 348.2 & 80.4 & 5.3 & 1.5 & 51.6 & $<0.2$ & 30 & 1.5 & 4.8 & 298.6 & $<1.0$ & $<2.0$ & 0.26 & $<0.0$ \\
\hline TBPR & 5 & OSU & & 0.01 & 0.21 & 0.07 & 0.06 & 0.46 & 214.2 & 87.2 & 2.7 & 1.9 & 35.2 & $<0.2$ & 2.4 & 2.2 & 6.6 & 220.9 & $<1.0$ & $<2.0$ & 0.37 & \\
\hline TBPR & 5 & WIS & & & & & & & & & & & & & & & & & & & & \\
\hline TBPR & 6 & OSU & & 0.03 & 0.23 & 0.04 & 0.06 & 0.54 & 704.6 & 25.9 & 3.1 & 1.6 & 7.3 & $<0.2$ & 2.9 & 1.2 & 5.0 & 72.1 & $<1.0$ & $\leq 2.0$ & .31 & 0.0 \\
\hline TBPA & 7 & OSU & & 0.01 & 0.07 & 0.05 & 0.05 & 0.33 & 640.0 & 36.8 & 6.1 & 1.7 & 3.0 & $<0.2$ & 2.7 & 1.8 & 3.1 & 147.2 & $<1.9$ & $<2.0$ & 0.33 & 0.0 \\
\hline TBPR & 7) & WIS & & 0.01 & 0.09 & 0.06 & 0.06 & 0.33 & 603.0 & & 5.4 & 2.0 & 3.3 & $<0.2$ & 2.4 & 1.1 & 3.2 & 165.1 & & $<2.0$ & & \\
\hline TBPA & 8 & OSU & & 0.01 & 0.14 & 0.04 & 0.09 & 0.27 & 345.1 & 44.4 & 7.8 & 1.7 & 8.2 & $<0.2$ & 7.4 & 1.4 & 5.8 & 28.5 & $<1.0$ & $\leq 2.0$ & 0.46 & 0.0 \\
\hline TBPA & 9 & $\overline{\mathrm{OSU}}$ & & 0.02 & 0.20 & 0.09 & 0.03 & & 299.4 & 41.2 & 30.6 & 1.4 & $<2.0$ & $<0.2$ & 2.4 & 1.9 & 4.6 & 43.5 & $<1.0$ & $<2.0$ & $<0.2$ & 0.8 \\
\hline TBPR & 9 & OSU & DUP & 0.02 & 0.21 & 0.09 & 0.03 & 0.36 & 327.2 & 40.0 & 30.7 & 1.1 & 2.4 & $<0.2$ & 1.6 & $\leq 0.6$ & 4.6 & 42.1 & $<1.0$ & $<2.0$ & 0.27 & \\
\hline TBPR & 10 & OSU & & 0.01 & 0.13 & 0.07 & 0.05 & 0.30 & 257.2 & 35.0 & 18.2 & 1.1 & 4.0 & $<0.2$ & 2.4 & 1.5 & 4.5 & 62.3 & $<1.0$ & $<2.9$ & 0.43 & 0.0 \\
\hline TBPA & 11 & OSU & & 0.01 & 0.13 & 0.19 & 0.03 & 0.20 & 278.1 & 33.8 & 16.2 & 2.4 & 2.9 & $<0.2$ & 2.0 & 2.0 & 4.3 & 80.9 & $<1.0$ & $<2.0$ & $<0.2$ & $\leq 0$. \\
\hline TBPA & 11 & WIS & & 0.01 & 0.15 & 0.19 & 0.03 & 0.15 & 334.0 & 19 & 16.6 & 3.0 & 2.8 & 0. & 1. & 1.2 & 4.0 & 77.9 & & $<2.0$ & & \\
\hline TBPA & 12 & OSU & & 0.02 & 0.13 & 0.05 & 0.08 & 0.34 & 77.3 & 45.9 & 4.4 & 3.5 & 6.9 & $<0.2$ & 4.7 & 2.3 & 6.3 & 64.6 & $\leq 1.0$ & $\leq 2.0$ & 0.54 & $\leq 0$. \\
\hline TBPA & 13 & OSU & & 0.03 & 0.22 & 0.05 & $\overline{0.12}$ & 0.29 & 126.6 & 36 & 3.6 & 1. & 8. & & 5.4 & 2.2 & 6.5 & 51.0 & $<1.0$ & $<2.0$ & .83 & $<0$. \\
\hline TBPR & 13 & OSU & DUP & 0.03 & 0.22 & 0.05 & 0.12 & 0.33 & 145.3 & 49.0 & 3.2 & 1.3 & 9.4 & $<0.2$ & 5.5 & $<0.6$ & 6.0 & 47.4 & $<1.0$ & $\leq 2.0$ & 0.74 & 0.0 \\
\hline TBPA & 14 & OSU & & 0.02 & 0.22 & 0.03 & 0.08 & 0.77 & 219.6 & 24.1 & 4.9 & 3.0 & 8.4 & $\leq 0.2$ & 3.2 & 1.5 & 6.2 & 12.9 & $<10$ & $\leq 2.0$ & 0.50 & $\leq 0$. \\
\hline TBPA] & 15 & OSU & & 0.02 & 0.27 & 0.02 & 0. & 0.39 & 273.0 & & 5.2 & 3.5 & & & 3.5 & 1.6 & 6.4 & & $\leq 1$. & $\leq 2.0$ & 40 & \\
\hline TBPA & 16 & OSU & & & & 0.05 & 0.08 & & 36 & & 8.8 & 3.9 & 7.7 & $<0$ & & & 5.1 & & & $<2.0$ & 0.46 & \\
\hline TBPR & 17 & OSU & & & & 0 & & & 355.2 & 35. & 7.6 & 2.8 & $<2.0$ & $<0.2$ & 2.1 & 1.5 & 6.8 & 50.6 & $<1.0$ & $<2.0$ & 0.39 & 0.0 \\
\hline TBPR & 18 & OSU & & 0.01 & 0.22 & 0.07 & & & & & & 2.4 & $\leq 2.0$ & $\leq 0.2$ & 2.2 & 1.8 & 3.7 & 43.4 & $\leq 1.0$ & $<2.0$ & 0.38 & 0.0 \\
\hline TBPR & 19 & OSU & & 0.0 & & 0. & 0.02 & & 122.5 & & 2.2 & 2.4 & 2.7 & & & & 6.6 & & & $<2$ & & 0.0 \\
\hline TBPA & 20 & $\mathrm{OSU}$ & & & 0.08 & & & & 212.7 & & 3. & 0.8 & 6.7 & $<0$. & & & & & $<1.9$ & & $<0.2$ & \\
\hline TBPR & 20 & $\overline{o s u}$ & DUP & & & & & & & & & & 6. & & & $<0.6$ & & 42 & $<1.0$ & $<2.0$ & $<0.2$ & 0.0 \\
\hline TBPR & 20 & WIS & & 0.01 & 0.08 & 0.08 & & & & & & & & & & 1.5 & & & & $\leq 2$ & & \\
\hline TBPR & 211 & osu & & & 0.18 & 0.07 & & & 143 & 24.2 & 23. & $\overline{1.3}$ & 7.3 & & & 0.8 & 8.8 & & $<1.0$ & $<2.0$ & 0.33 & 0.0 \\
\hline PR! & 221 & OSU & & 0.02 & 0.16 & 0.04 & & & & & 25.7 & 0.9 & 6.1 & $<0.2$ & 2. & 1.1 & 5.4 & 44.0 & $<1.0$ & $\leq 2.0$ & 0.30 & $<0.0$ \\
\hline TBPR & 23 & OSU & & 0.02 & 0.17 & 0.06 & & & & & & & & & & & & 60.3 & $<1.0$ & $<2.9$ & 0.46 & \\
\hline TBPR & 23 & OSU & DUP & 0.0 & 0.1 & 0.06 & 0.03 & & & 21.0 & 26 & 1. & 8.2 & & & & & & & & & \\
\hline $\mathrm{PB}$ & 24 & OSU & & 0.01 & & & & & & & 23. & 1 & 14.3 & $<0$. & 4. & 0 & 4.1 & 72. & $<1,0$ & $<2.0$ & 0.30 & \\
\hline$\overline{\mathrm{PR}}$ & 251 & OSU & & 0.0 & 0.0 & & & & & & & 0 & 6.7 & $\leq 0$ & 2. & $<0.6$ & 4.4 & & $\leq 1.0$ & 52.0 & $<0,2$ & $\leq 0.1$ \\
\hline & 26 & OSU & & 0.01 & 0.18 & 0.08 & 0.0 & 0.44 & & 20 & 20.0 & 1 & 6. & & & & & & & & 0.25 & $<0$. \\
\hline $\mathrm{PR}$ & 27 & OSU & & & & & & & & & 22.2 & & & $<0.2$ & 2.9 & $<0.6$ & & & & $<2.0$ & $<0.2$ & \\
\hline $\mathrm{PR}$ & 27 & osu & DUP & & & & & & & & & 1.1 & 11.3 & $<0.2$ & & $<0.6$ & 4.8 & & $<1.0$ & $<2.0$ & 0.21 & \\
\hline Ph & 28 & OSU & & 0.01 & 0.19 & 0.03 & 0.06 & 0.19 & 1685.4 & 26.2 & 31.5 & 1.3 & 9.1 & & 4. & 0.7 & 3.2 & 56.8 & $<1.0$ & $\leq 2,0$ & 0.25 & 0.0 \\
\hline PR & 29 & OSU & & & & & 0.05 & 0.59 & 1398.9 & 38.3 & 36.9 & 1.1 & 11.5 & $<0.2$ & 3.3 & $\leq 0.6$ & 5.9 & 177.0 & 1.0 & $\leq 2.9$ & 0.21 & 0.0 \\
\hline & & & & & & & & & & & 22.0 & & & & & 0.6[ & 3.4 & & & & & \\
\hline
\end{tabular}


Table A. 3 cont.

\begin{tabular}{|c|c|c|c|c|c|c|c|c|c|c|c|c|c|c|c|c|c|c|c|c|c|c|}
\hline & & & & LEAF & LEAF & LEAF & LEAF & LEAF & LEAF & LEAF & LEAF & LEAF & LEAF & LEAF & LEAF & LEAF & LEAF & LEAF & LEAF & LEAF & LEAF & LEAF \\
\hline SITEI & & & & $P$ & $\mathrm{k}$ & $\mathrm{Ca}$ & $\mathrm{Mg}$ & $\mathrm{N}$ & $\mathrm{Na}$ & $\mathrm{Fe}_{\mathrm{e}}$ & $M n$ & B & $\mathrm{Pb}$ & $\mathrm{Cd}$ & $\mathrm{Ni}$ & $\mathrm{Cr}$ & $\mathrm{Cu}$ & $\mathrm{Zn}$ & $\mathrm{Hg}$ & As & Mo & $\mathrm{Se}$ \\
\hline STUDY & ID & $\mathrm{LAB}$ & TYP & $(\%)$ & $(\%)$ & (\%) & $(\%)$ & (\%) & $(\mathrm{ug} / \mathrm{g})$ & $(\mathrm{ug} / \mathrm{g})$ & $(\mathrm{ug} / \mathrm{g})$ & $(u g / g)$ & $(u g / g)$ & $(u g / g)$ & $(u g / g)$ & $(u g / g)$ & $(u g / g)$ & $(u g / g)$ & $(u g / g)$ & $(u g / g)$ & $(u g / g)$ & $(u g / g)$ \\
\hline TBPA & 1 & OSL & & 0.03 & 0.04 & 0.13 & 0.05 & 1.24 & 103.5 & 107.8 & 16.1 & 7.7 & 7.1 & $<0,2$ & 18 & 1,3 & 2.2 & 56.8 & $<1.0$ & $<2.0$ & 0.74 & \\
\hline TBPR & & OSL & & 0.03 & 0.04 & 0.18 & 0.06 & 0.92 & 52.6 & 151.4 & 6.1 & 3.7 & 107.0 & $<0.2$ & 3.3 & 2.3 & 9.1 & 450.1 & $<1.0$ & $<2.0$ & 0.82 & 0.04 \\
\hline TBPR & 2 & OSL & DUI & & & & & & & & & & & & & & & & & & & \\
\hline IBPA & 2 & WIS & & 0.03 & 0.07 & 0.32 & 0.13 & $\begin{array}{l}0.79 \\
116\end{array}$ & $\frac{73.0}{836}$ & $=\frac{104.6}{254 ?}$ & $\frac{7.8}{106}$ & $\frac{6.9}{6.5}$ & $\begin{array}{r}44.6 \\
1097\end{array}$ & $<0.2$ & $\frac{3.6}{32}$ & $\frac{1.7}{1.4}$ & $\frac{5.3}{5.7}$ & $\frac{406.1}{509.1}$ & $\langle 1.0$ & $\frac{<2.9}{<2.0}$ & 0.91 & \\
\hline TBPA & 3 & OSU & & 0.04 & 0.06 & 0.22 & 0.10 & 1.16 & $\begin{array}{r}83.6 \\
1109\end{array}$ & $\frac{254.2}{118.3}$ & $\begin{array}{r}0.0 \\
9.6\end{array}$ & $\frac{1.5}{8.2}$ & $\frac{10.1}{15.3}$ & $<0.2$ & 3.5 & 1.1 & 3.2 & 329.2 & $<1.0$ & $<2.0$ & 0.33 & 0.04 \\
\hline TBPA & 4 & OSL & & 0.04 & 0.05 & 0.17 & 0.07 & 0.75 & $\frac{110.9}{80.2}$ & 66.4 & 6.3 & 6.4 & 22.4 & $<0.2$ & 2.1 & 1.1 & 3.1 & 297.2 & $<1.0$ & $\frac{<.0}{<2.0}$ & $\frac{0.03}{0.64}$ & 0,08 \\
\hline TBPR & 5 & OSL & & -0.02 & 0.03 & $\frac{0.32}{0.38}$ & $\begin{array}{l}0.09 \\
0.09\end{array}$ & $\begin{array}{l}0.13 \\
0.56\end{array}$ & 106.0 & 95.6 & 7.5 & 5.7 & 22.9 & $<0.2$ & 2.1 & 1.4 & 3.2 & 377.9 & & $<20$ & & 0.08 \\
\hline$\frac{1 B P H}{T B P A}$ & $\frac{5}{6 !}$ & $\frac{\mathrm{WIS}}{\mathrm{OSL}}$ & & $0.0 \leq$ & 0,03 & & & & & & & & & & & & & & & & & \\
\hline TBPA & 7 & OSL & & 0.04 & 0.05 & 0.17 & 0.07 & & 97.1 & 79.1 & 5.7 & 2.9 & 4.5 & $<0.2$ & 2.8 & 1.2 & 2.4 & 148.3 & $\leq 1.2$ & $<2.5$ & 0.96 & \\
\hline TBPA & 7 & WIS & & & & & & & & & & & & & & & & & & & & \\
\hline TBPR & 8 & OSU & & 0.03 & 0.03 & 0.21 & 0.13 & 0.71 & 118.6 & 102.5 & 32.2 & 6.5 & 5.3 & $<0.2$ & 3.4 & 1.4 & 2.7 & 41.2 & $<1.0$ & $<2.0$ & 2.80 & 0.15 \\
\hline TBPA & 9 & OSL & & 0.03 & 0.04 & 0.38 & 0.04 & 0.83 & 151.9 & 116.1 & 62.7 & 7.8 & 8.0 & $<0.2$ & 3.4 & 1.9 & 4.3 & 81.4 & $\leq 1.9$ & $<2.0$ & 0.41 & 0.11 \\
\hline TBPR & 9 & OSL & DUP & & & & & & & & & & & & & & & & & & & \\
\hline TBPR & 10 & OSU & & & & & & & & & & & & & & & & & & & & \\
\hline TBPR & 11 & OSU & & 0.05 & 0.04 & 0.38 & 0.04 & & 162.1 & 99.0 & 40.4 & 11.7 & 9.8 & $<0.2$ & 9.4 & 1.4 & 3.4 & 86.9 & $<1.0$ & $<2.0$ & 0.61 & \\
\hline TBPR & 11 & WIS & & & & & & & & & & & & & & & & & & & & \\
\hline TBPA & 12 & OSL & & 0.05 & 0.07 & 0.30 & 0.16 & 0.78 & 135.1 & 894.6 & 24.7 & 11.1 & 33.6 & 0.2 & 11.8 & 12.7 & 29.7 & 136.5 & $\leq 1.0$ & $<2.0$ & 1.20 & \\
\hline TBPA & 13 & OSL & & 0.05 & 0.05 & 0.29 & 0.15 & 0.71 & 117.3 & 99.3 & 11.4 & 6.2 & 4.0 & $<0.2$ & 3.7 & 1.5 & 2.7 & 87.7 & $\leq 1.0$ & $<2.0$ & 3.40 & \\
\hline TBPA & 13 & OSL & DUP & & & & & & & & & & & & & & & & & & & \\
\hline TBPA & 14 & OSL & & 0.04 & 0.07 & 0.21 & 0.14 & 0.82 & 92.1 & 65. & 12.1 & 6.8 & 5.9 & $<0.2$ & 3.6 & 1.5 & 2.6 & 30.7 & $<1.0$ & $\leq 2.0$ & 1.50 & 0.13 \\
\hline TBPA & 15 & OSL & & 0.04 & 0.04 & 0.18 & 0.16 & & 132.8 & 115.4 & 66.3 & 10.1 & 4.6 & $<0.2$ & 4.1 & 1.6 & 3.0 & 30.3 & $\leq 1.2$ & $\leq 2.4$ & 1.40 & \\
\hline TBPA & 16 & OSU & & 0.03 & 0.04 & 0.38 & 0.14 & & 128.5 & 89.4 & 42.4 & 10.7 & 8.3 & $<0.3$ & 7.2 & 1.2 & 4.2 & 47.3 & $<1.4$ & $<2.8$ & 2.30 & \\
\hline TBPR & 17 & OSU & & & & & & & & & & & & & & & & & & & & \\
\hline TBPA & 18 & OSL & & 0.05 & 0.05 & 0.36 & 0.06 & & 133.5 & 96.6 & 20.4 & 9.7 & 3.9 & $<0.2$ & 2.2 & 1.6 & 3.5 & 80.0 & $<1.1$ & $\leq 2.3$ & 1.10 & \\
\hline TBPR & 19 & OSU & & & & & & & & & & & & & & & & & & & 030 & \\
\hline TBPA & 20 & $\mathrm{OSL}$ & & 0.02 & 0.03 & 0.27 & 0.04 & 0.78 & 167.1 & 114.6 & 8.3 & 5.6 & 6.9 & $<0.2$ & 4.1 & 1.6 & 4.0 & 82.6 & $<1.9$ & $\leq 2.0$ & 0.39 & \\
\hline TBPR & 20 & OSL & DUI & & & & & & & & & & & & & & & & & & & \\
\hline TBPA & 20 & WIS & & & & & & & & & & & & & & & & & & & & \\
\hline TBPA & 21 & OSU & & 0.04 & 0.04 & 0.23 & 0.15 & 0.81 & 163.7 & 102.5 & 35.4 & 7.1 & 9.1 & $<0.2$ & 4.4 & 1.3 & 3.9 & 207.8 & $<1.0$ & $<2.0$ & 0.88 & 0.13 \\
\hline TBPA & 22 & OSL & & & & & & & & & & & & & & & & & & & & \\
\hline TBPA & 23 & OSL & & & & & & & & & & & & & & & & & & & & \\
\hline TBPR & 23 & OSL & DUP & & & & & & & & & & & & & & & & & & & \\
\hline TBPR & 24 & $\overline{O S U}$ & & & & & & & & & & & & & & & & & & & & \\
\hline TBPA & 25 & OSU & & & & & & & & & & & & & & 28 & & & & & & \\
\hline TBPA & 26 & OSU & & 0.05 & 0.04 & 0.15 & 0.15 & & 77.9 & 125.9 & 18.9 & 6.8 & 4.3 & $\leq 0.2$ & 6.1 & 2.8 & 3.7 & 40.3 & $\leq 1.2$ & & 1.30 & \\
\hline TBPR & 27 & $\mathrm{OSU}$ & 010 & & & & & & & & & & & & & & & & & & & \\
\hline$\frac{T B P R}{\text { TBPA }}$ & $\frac{27}{28}$ & $\frac{\mathrm{OSU}}{\mathrm{OSU}}$ & DUF & & & & & & & & & & & & & & & & & & & \\
\hline TBPR & 29 & OSU & & & & & & & & & & & & & & & & & & & & \\
\hline TBPR & 30 & OSU & & & & & & & & & & & & & & & & & & & & \\
\hline
\end{tabular}


Table A. 3 cout.

\begin{tabular}{|c|c|c|c|c|c|c|c|c|c|c|c|c|c|c|c|c|c|c|c|c|c|c|}
\hline & & & & SEED & SEED & SEED & SEED & SEED & SEED & SEED & SEED & SEED & SEED & \begin{tabular}{|l|} 
SEED \\
\end{tabular} & SEED & SEED & SEED & SEED & SEED & SEED & SEED & SEED \\
\hline SITEI & & & & $\mathrm{P}$ & $k$ & $\mathrm{Ca}$ & $\mathrm{Mg}$ & $N$ & $\mathrm{Na}$ & $\mathrm{Fe}$ & $\mathrm{Mn}$ & B & $\mathrm{Pb}$ & $\mathrm{Cd}$ & $\mathrm{Ni}$ & $\mathrm{Cr}$ & $\mathrm{Cu}$ & $\mathrm{Zn}$ & $\mathrm{Hg}$ & As & Mo & $\mathrm{Se}$ \\
\hline STUDY & 10 & $\angle A B$ & TYPE & (\%) & (\%) & $(\%)$ & $(\%)$ & (\%) & $(\mathrm{ug} / \mathrm{g})$ & $(\mathrm{ug} / \mathrm{g})$ & $(\mathrm{ug} / \mathrm{g})$ & $(u g / g)$ & $(u g / g)$ & $(u g / g)$ & $(u g / g)$ & $\langle u g / g)$ & $(u g / g)$ & $(\mathrm{ug} / \mathrm{g})$ & $(u g / g)$ & $(u g / g)$ & $(u g / g)$ & $(u g / g)$ \\
\hline TBPA & 1 & OSU & & & & & & & & & & & & & & & & & & & & \\
\hline TBPA & 2 & OSU & & 0.14 & 0.12 & 0.09 & 0.09 & 2.14 & 141.6 & 148.2 & 6.9 & 6.1 & 10.2 & $<0.2$ & 2.9 & 1.6 & 10.5 & 278.0 & $<1.0$ & $<2.0$ & 1.00 & 0.16 \\
\hline TBPR & 2 & OSU & $\overline{\text { DUP }}$ & & & & & & & & & & & & & & & & & & & \\
\hline TBPR & 2 & WIS & & & & & & & & & & & & & & & & & & & & \\
\hline$\frac{\text { TBPR }}{\text { TBPP }}$ & $\frac{3}{4}$ & $\frac{\mathrm{OSU}}{\mathrm{OSU}}$ & & 0.09 & 0.06 & 0.09 & 0.06 & 1.54 & 192.9 & 125.3 & 9.8 & 6.0 & 202 & $<02$ & 30 & 08 & 75 & 3632 & 50 & 20 & 055 & 024 \\
\hline TBPA & $\begin{array}{l}5 \\
5\end{array}$ & OSU & & $\ldots$ & 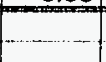 & & 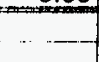 & & 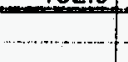 & $-\quad$ & & & 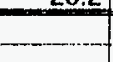 & 4 & & & & & & & 0.00 & 0.24 \\
\hline$\frac{1 B P H}{T B P A}$ & $\frac{5}{6}$ & $\frac{\text { WIS }}{\text { OSU }}$ & & 0.17 & 0.10 & 0.10 & 0.08 & 1.83 & 146.3 & 110.9 & 28.4 & 5.9 & 5.0 & $<0.2$ & 2.0 & 1.1 & 10.0 & 114.6 & $<1.0$ & $<2.0$ & $\overline{0.79}$ & 0.13 \\
\hline TBPR & 7 & OSU & & 0.14 & 0.08 & 0.09 & 0.10 & 1.36 & 154.1 & 115.7 & 9.3 & 4.7 & 10.8 & $<0.2$ & 2.6 & 1.0 & 8.0 & 203.8 & $<1.0$ & $<2.0$ & 0.93 & \\
\hline TBPR & 7 & WIS & & 0.12 & 0.10 & 0.10 & 0.09 & 1.23 & 179.0 & 92.4 & 8.7 & 4.6 & 4.0 & $<0.2$ & 2.1 & 0.9 & 10.1 & 245.8 & & $<2.0$ & & \\
\hline TBPR & 8 & osu & & & & & & & & & & & & & & & & & & & & \\
\hline TBPR & 9 & OSU & & 0.11 & 0.05 & 0.11 & 0.04 & & 391.8 & 277.3 & 18.1 & 7.1 & 6.5 & $<0.5$ & 2.9 & $<1.4$ & 9.7 & 96.7 & $<2.3$ & $<4.5$ & 0.73 & \\
\hline TBPR & 9 & osu & DUP & & & & & & & & & & & & & & & & & & & \\
\hline TBPR & 10 & OSU & & & & & & & & & & & & & & & & & & & & \\
\hline TBPA & 11 & OSU & & 0.07 & 0.04 & 0.13 & 0.03 & & 183.1 & 92.7 & 11.5 & 4.6 & 6.4 & $<0.4$ & 2.0 & $\leq 1.2$ & 11.9 & 102.0 & $<2.1$ & $<4.2$ & 0.92 & \\
\hline TBPR & 11 & WIS & & & & & & & & & & & & & & & & & & & & \\
\hline TBPR & 12 & & & & & & & & & & & & & & & & & & & & & \\
\hline TBPR & 13 & OSU & & 0.12 & 0.06 & 0.09 & 0.11 & 1.35 & 340.8 & 195.2 & 10.1 & 8.2 & 4.7 & $<0.2$ & 3.5 & 1.2 & 12.0 & 124.7 & $<1.9$ & $<2.0$ & 2.60 & \\
\hline TBPA & 13 & OSU & DUP & & & & & & & & & & & & & & & & & & & \\
\hline TBPR & 14 & OSU & & 0.14 & 0.10 & 0.09 & 0.15 & & 119.3 & 142.1 & 11.8 & 18.4 & 6.2 & $\leq 0.3$ & 7.0 & 1.4 & 10.6 & 66.6 & $<1.5$ & $\leq 2.9$ & 1.50 & \\
\hline TBPA & 15 & OSU & & & & & & & & & & & & & & & & & & & & \\
\hline$\frac{T B P A}{T B P A}$ & $\frac{16}{171}$ & osu & & & & & & & & & & & & & & & & & & & & \\
\hline TBPA & 18 & osu & & 0.12 & 0.06 & 0.11 & 0.07 & 1.40 & 200.9 & 214.3 & 11.6 & 4.5 & 4.7 & $<0.2$ & 2.8 & $<0.6$ & 11.8 & 146.2 & $<1.0$ & $<20$ & 140 & 059 \\
\hline TBPR & 19 & osu & & 0.11 & 0.10 & 0.09 & 0.06 & 1.87 & 590.6 & 179.5 & 8.9 & 8.3 & 7.9 & $\leq 0.2$ & 4.2 & 1.6 & 19.6 & 149.2 & $\leq 1.0$ & $<2.0$ & $\frac{0.404}{0.54}$ & 0.33 \\
\hline TBPR & 20 & osu & & & & & & & & & & & & & & & & & & & & \\
\hline TBPR & 20 & osu & DUP & & & & & & & & & & & & & & & & & & & \\
\hline TBPR & 201 & WIS & & & & & & & & & & & & & & & & & & & & \\
\hline TBPR & 21 & OSU & & 0.08 & 0.04 & 0.07 & 0.03 & 1.24 & 161.9 & 95.5 & 21.5 & 2.6 & 3.4 & $<0.2$ & 2.1 & $<0.6$ & 8.0 & 94.6 & $\angle 1.0$ & $<2.0$ & 0.97 & 0.15 \\
\hline TBPRI & 221 & क्SU & & & & & & & & & & & & & & & & & & & & \\
\hline TBPR & 231 & osu & & & & & & & & & & & & & & & & & & & & \\
\hline TBPR & 23 & & DUP & & & & & & & & & & & & & & & & & & & \\
\hline TBPA & 24 & osu & & & & & & & & & & & & & & & & & & & & \\
\hline TBPA & 25.0 & 250 & & & & & & & & & & & & & & & & & & & & \\
\hline TBPR & 260 & OSU & & 0.24 & 0.14 & 0.12 & 0.10 & 1.95 & 103.4 & 83.3 & 35.7 & 6.4 & 3.4 & $\leq 0.2$ & 2.1 & $\leq 0.6$ & 9.8 & 97.8 & $<1.0$ & $<2.0$ & 0.67 & 0.12 \\
\hline TBPR & 270 & SSU & & & & & & & & & & & & & & & & & & & & \\
\hline TBPR & $27 \mathrm{C}$ & ssu & DUP & & & & & & & & & & & & & & & & & & & \\
\hline TBPA & 280 & SSU & & & & & & & & & & & & & & & & & & & & \\
\hline TBPA & $29 C$ & SUI & & & & & & & & & & & & & & & & & & & & \\
\hline & $30] \mathrm{C}$ & SU & & & & & & & & & & & & & & & & & & & & \\
\hline
\end{tabular}

